Analysis of a linearization scheme for an interior penalty discontinuous Galerkin method for two phase flow in porous media with dynamic capillarity effects

Non Peer-reviewed author version

KARPINSKI, Stefan; POP, Sorin \& Radu, Florin Adrian (2017) Analysis of a linearization scheme for an interior penalty discontinuous Galerkin method for two phase flow in porous media with dynamic capillarity effects. In: INTERNATIONAL JOURNAL FOR NUMERICAL METHODS IN ENGINEERING, 112(6), p. 553-577.

DOI: $10.1002 / \mathrm{nme} .5526$

Handle: http://hdl.handle.net/1942/25329 


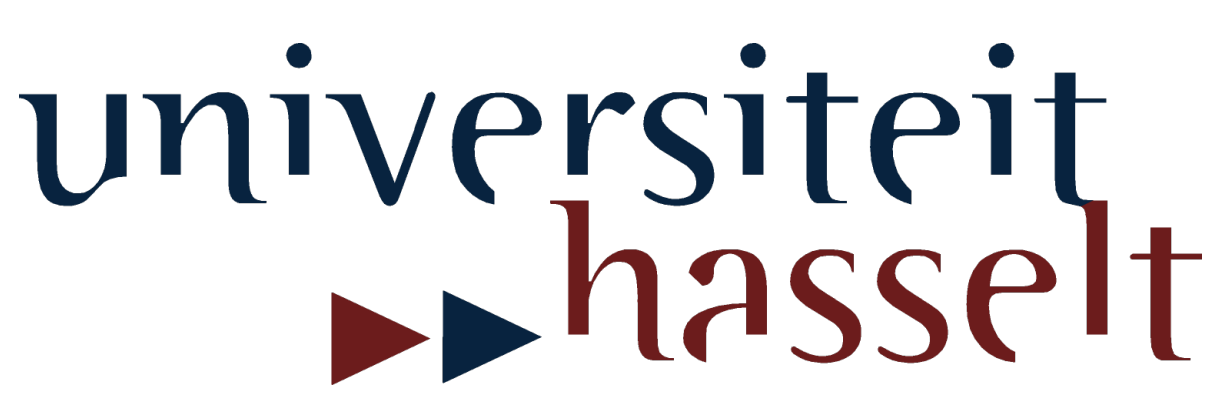

UHasselt Computational Mathematics Preprint Series

Analysis of a linearization scheme for an interior penalty discontinuous Galerkin method for two phase flow in porous media with dynamic capillarity effects

Stefan Karpinski, Iuliu Sorin Pop, Florin A. Radu

UHasselt Computational Mathematics Preprint Nr. UP-16-05

November 17, 2016 


\title{
Analysis of a linearization scheme for an interior penalty discontinuous Galerkin method for two phase flow in porous media with dynamic capillarity effects
}

\author{
Stefan Karpinski ${ }^{* 1,2}$, Iuliu Sorin Pop ${ }^{2,3}$ and Florin Adrian $\mathrm{Radu}^{3}$ \\ ${ }^{1}$ ESPRiT Engineering GmbH \\ ${ }^{2}$ Faculty of Sciences, Hasselt University \\ ${ }^{3}$ Dept. of Mathematics, University of Bergen
}

\begin{abstract}
SUMMARY
Abstract We present a linearization scheme for an interior penalty discontinuous Galerkin method for two-phase porous media flow model which includes dynamic effects in the capillary pressure. The fluids are assumed immiscible and incompressible, and the solid matrix non-deformable. The physical laws are approximated in their original form, without using the global or complementary pressures. The linearization scheme does not require any regularization step. Furthermore, in contrast with Newton or Picard methods, there is no computation of derivatives involved. We prove rigorously that the scheme is robust and linearly convergent. Numerical results, including examples in realistic, heterogeneous media are presented to sustain the applicability of the scheme. Copyright (C) 2016 John Wiley \& Sons, Ltd.
\end{abstract}

KEY WORDS: two-phase flow; heterogenous porous media; dynamic capillary pressure; interior penalty discontinuous Galerkin method; linearization method; convergence analysis; L-scheme

\section{INTRODUCTION}

Flow and transport processes in porous media are of high interest in many different fields of application. In this sense we mention the geological $\mathrm{CO}_{2}$-storage [26], environmental pollution [31], designing diapers [10], or filters. The mathematical models describing such phenomena are, in general, highly non-linear and demanding in terms of the numerical solution. To resolve the nonlinearities, one usually uses the Newton or Picard methods, see e.g. [3, 6, 24], combination of them [21, 23], or iterative IMPES [18, 19]. The Newton-scheme shows a local convergence with quadratic order, however, if the initial guess is cloes enough to the solution, while Picard-iterations are more robust but only linear convergent. For designing Newton or Picard methods for degenerate problems as appearing in porous media flows, on needs to include a regularization step. We mention also the

\footnotetext{
*Correspondence to: stefan.karpinski@esprit-engineering.de
} 
semi-smooth Newton method [20], which is a valuable alternative to Newton's method especially for multi-component flow. Its drawback is the relatively high implementation cost.

As mentioned, the Newton-scheme has a high convergence order, which makes it very attractive for solving nonlinear problems. However, two aspects have to be taken into account. First, one has to calculate the Jacobian matrix or at least a proper approximation of it for any iteration step, which, in general, is computationally expensive. Second, to guarantee the convergence of the iteration, the initial guess should be close enough to the solution. This aspect is analyzed e.g. in [27] for the mixed finite element discretization of nonlinear elliptic problems, where the difference between the initial guess and the exact solution should be of order $h^{d}(h$ being the mesh size and $d$ the dimension of the domain). For parabolic partial differential equations, a straightforward option is the solution obtained at the previous time-step. Nevertheless, to make sure that this is indeed close enough, the time-step has to be chosen sufficiently small, again of order $h^{d}$. This restriction becomes more severe when degenerate parabolic problems are considered. In this case, in locations where one of the phases is not present, the permeability of this phase vanishes. This has two immediate consequence for the numerical approximation, as the Jacobian becomes singular, leading to ill-conditioned linear systems. To avoid this, one needs to regularize the problem, i.e. to consider perturbations of it assuring that the problem remains non-degenerate. This is an additional source of errors in the system. More important, the restriction on the time step becomes more severe in this case, as it also involves the small regularization parameter as well (see [32]; similar issues appear for reactive flow models with non-Lipschitz rates [31].

The above has motivated the linearization schemes proposed in $[23,28,29,30,34,36]$ for the finite element, finite volume or the mixed finite element discretization of porous media flow models. The idea of the linearization scheme is to add an additional term in the form of

$$
L \cdot(\text { Solution_Current_Iteration - Solution_Old_Iteration }),
$$

with $L$ being a parameter that has to be chosen sufficiently large. The robustness of such schemes (also called $L$-schemes) for standard porous media flow models is proved in the papers mentioned above. Although the convergence is linear, the $L$-scheme may even become faster than the Newton method as it does not require to compute derivatives, while the linear systems to be solved within each iteration are better conditioned (see [23], where also the possibility to combine the $L$-scheme with the Newton iteration has also been discussed) and may even involve the same matrix for the linear algebraic system, which offers the possibility to compute its factorization only once per time step. Moreover, the $L$-scheme does not involve any regularization step.

Inspired by the results above, we propose a linear convergent iterative scheme for the two-phase flow in porous media. Here we consider a non-standard mathematical model of pseudo parabolic type, as it involves a dynamic term in the phase pressure difference - saturation relationship (the dynamic capillarity), see [13, 14]. For this model a discontinuous Galerkin (dG) method discretization is coupled with the backward Euler time stepping. For details we refer to [16], where the convergence of the scheme is proved assuming that the capillary damping term is 
constant. Finally, the model formulation adopted here does not involve any global or complementary pressure, as done in $[29,30]$. We prove give a rigorous convergence proof for the $L$-scheme, and provide numerical experiments confirming the theoretical findings. These experiments also include heterogeneous media. To our knowledge, this is the first time when such a scheme is tested in the case of a heterogeneous medium.

The structure of the paper is as follows. In Section 2, we introduce the mathematical model for two-phase flow with dynamic capillarity, in Section 3 we present the numerical solution strategy and approximation with an interior penalty discontinuous Galerkin approximation. Section 4 is devoted to the convergence analysis of the presented linearization scheme. In order to show the versatility of the scheme, relevant numerical examples are presented in Section 5. Both homogeneous and heterogeneous porous media are considered.

\section{MATHEMATICAL MODEL FOR TWO-PHASE FLOW IN POROUS MEDIA WITH DYNAMIC CAPILLARITY}

In this section we present the mathematical model and give the notion of a weak solution. Also, the working assumptions are provided.

Governing equations We consider two incompressible and immiscible fluids flowing through a non-deformable porous medium. The medium occupies the bounded polygonal domain $\Omega \subset \mathbb{R}^{d}$, $d=2,3$. Its boundary is denoted by $\Gamma$. The flow takes place in the time interval $(0, T]$ with given $T>0$. Under these assumptions, mass conservation holds for each phase, i.e. for each phase $\alpha$ one has

$$
\partial_{t}\left(S_{\alpha} \phi \rho_{\alpha}\right)+\nabla \cdot\left(\rho_{\alpha} \mathbf{u}_{\alpha}\right)=q_{\alpha}
$$

Here $\alpha \in\{n, w\}$ stands for non-wetting and wetting phase, respectively. Further, $\phi$ denotes the porosity of the medium, $\rho_{\alpha}$ the fluid phase densities, $q_{\alpha}$ the volumetric sources or sinks, and $\mathbf{u}_{\alpha}$ the fluid phase Darcy velocities. The latter are given by the Darcy laws

$$
\mathbf{u}_{\alpha}=-\lambda_{\alpha}\left(S_{w}\right) K \nabla p_{\alpha}
$$

where, $p_{\alpha}$ are the phase pressures, $K$ the intrinsic permeability tensor, and $\lambda_{\alpha}=\frac{k_{r, \alpha}}{\mu_{\alpha}}$ the fluid mobility functions with relative permeabilities $k_{r, \alpha}$ and dynamic viscosities $\mu_{\alpha}$. The nonlinear functions $k_{r, \alpha}$ are determined experimentally and therefore assumed known here. We refer to [26] for more details regarding the above equations and modeling of two-phase flow in porous media.

Closure relationships The four equations above, (1)-(2), are involving six unknown quantities: $S_{\alpha}, p_{\alpha}, \mathbf{u}_{\alpha}$. To close the system, one needs two additional equations. The first follows from the 
assumption that only two fluid phases are encountered in the system. this gives

$$
S_{w}+S_{n}=1
$$

The second equation relates the phase pressure difference to the saturation of (say) the wetting phase

$$
p_{c}:=p_{n}-p_{w}=p_{c}\left(S_{w}, \partial_{t} S_{w}\right)=p_{c, e q}\left(S_{w}\right)-\tau\left(S_{w}\right) \partial_{t} S_{w}=p_{c, e q}\left(S_{w}\right)-\partial_{t} T\left(S_{w}\right)
$$

In the above, $p_{c, e q}$ is the capillary pressure at equilibrium, and $\tau\left(S_{w}\right)$ is a material depending term, often called capillary damping coefficient; its primitive is denoted by $T(\cdot)$. Observe that it is common in the literature to leave the $\partial_{t} S_{w}$ term out in (4), and reduce it to a nonlinear relationship between $p_{c}$ and $S_{w}$. Such models are called here equilibrium models. The $\tau\left(S_{w}\right)$-term is accounting for dynamic capillarity effects in the model, and it is motivated by experimental results like the ones in e.g, [9]. There saturation overshoot effects have been observed when letting water infiltrate into a homogeneous porous column that is initially dry. Such saturation profiles are ruled out in standard, equilibrium models, as their solution is satisfying a maximum principle. We refer to [12, 13, 14] for details on the origin and necessity of the dynamic capillarity and on the modelling.

Primary variables The six-equation model (1)-(4) can be reduced to the following three-equation system:

$$
\begin{aligned}
-\partial_{t}\left(S_{w} \phi\right)-\nabla \cdot\left(\lambda_{n}\left(S_{w}\right) K \nabla p_{n}\right) & =q_{n}, \\
\partial_{t}\left(S_{w} \phi\right)-\nabla \cdot\left(\lambda_{w}\left(S_{w}\right) K\left(\nabla p_{n}-\nabla p_{c}\right)\right) & =q_{w}, \\
p_{c}=p_{c, e q}\left(S_{w}\right)-\tau\left(S_{w}\right) \partial_{t} S_{w} & =p_{c, e q}\left(S_{w}\right)-\partial_{t} T\left(S_{w}\right) .
\end{aligned}
$$

In the above, the following variables have been chosen as primary: $p_{n}, S_{w}$, and $p_{c}$. Note that, for readability, below we still use $p_{w}=p_{n}-p_{c}$, although it is a secondary variable. A similar choice of the primary variables is made in $[24,17]$.

Initial and boundary conditions To close the system, we prescribe the following initial and boundary conditions:

$$
\begin{array}{lrl}
\text { For all } x \in \Omega \text { and at } t=0: & S_{w}(x, 0)=s^{0}(x) . & \\
\text { For all } x \in \Gamma_{D} \text { and } t \in[0, T]: & p_{c}(x, t)=p_{c}^{D}(x), \quad p_{n}(x, t)=p_{n}^{D}(x) .
\end{array}
$$

Observe that extending $\left(5_{3}\right)$ up to the boundary $\Gamma$ provides boundary data for $S_{w}$ as well.

For simplicity we only consider here Dirichlet boundary conditions. Moreover, the boundary values are assume constant in time. At the expense of additional technical aspects it is possible to extend these results so that other types of conditions are included.

Notations In general, solutions in classical sense are not available. We here we consider weak solutions, whose definition is involving abstract functional spaces. The following notations are common in the functional analysis/partial differential equations. For $1 \leq p<\infty, L^{p}(\Omega)$ is the space of $p$-integrable functions (in the sense of Lebesgue), while the elements of $L^{\infty}(\Omega)$ are essentially bounded functions; the corresponding norms are $\|\cdot\|_{L^{p}(\Omega)} \cdot\|\cdot\|$ stands for the $L^{2}$ norm. $\|\cdot\|_{W^{k, p}(\Omega)}$ denotes the standard norm on $W^{k, p}(\Omega)$, the space of functions admitting $L^{p}$ weak derivatives up to 
order $k$. For simplicity, we represent $W^{k, 2}(\Omega)$-norm with $\|\cdot\|_{\Omega, k}$ and use the notation $H^{k}(\Omega)$ for $W^{k, 2}(\Omega) . H_{0}^{k}(\Omega)$ denotes the space of $H^{k}(\Omega)$ functions that vanish on the boundary (in the sense of traces). With $X$ being any Banach space, $L^{2}(0, T ; X)$ denotes the space of Bochner-integrable, $X$-valued function on $[0, T]$, while $H^{1}(0, T ; X)$ denotes the space of functions in $L^{2}(0, T ; X)$ with a weak time-derivative in the same space. Finally, the boundary values should be interpreted in the sense of traces on $\Gamma$, which will lie in spaces like $L^{p}(\Gamma), H^{k}(\Gamma)$, etc. In particular, by $H^{\frac{1}{2}}(\Gamma)$ we mean the traces on $\Gamma$ of $H^{1}(\Omega)$ functions.

Weak formulation A weak solution to (5)-(7) solves

\section{Problem 1 (Weak formulation)}

Find the triple $\left(S_{w}, p_{n}, p_{c}\right)$ s.t. $S_{w} \in H^{1}\left([0, T], H^{1}(\Omega)\right), \quad S_{w}=s^{0} \quad$ at $t=0, \quad p_{n}-p_{n}^{D} \in$ $L^{2}\left([0, T], H_{0}^{1}(\Omega)\right), p_{c}-p_{c}^{D} \in L^{2}\left([0, T], H_{0}^{1}(\Omega)\right)$, and for all $\psi_{p} \in H_{0}^{1}(\Omega), \psi_{s} \in H_{0}^{1}(\Omega)$, and almost every $t \in[0, T]$ it holds

$$
\begin{aligned}
-\int_{\Omega} \partial_{t} S_{w} \phi \psi_{p}+\int_{\Omega} \lambda_{n}\left(S_{w}\right) K\left(\nabla p_{n}\right) \cdot \nabla \psi_{p} & =\int_{\Omega} q_{n} \psi_{p} \\
\int_{\Omega} \partial_{t} S_{w} \phi \psi_{p}+\int_{\Omega} \lambda_{w}\left(S_{w}\right) K\left(\nabla p_{n}-\nabla p_{c}\right) \cdot \nabla \psi_{p} & =\int_{\Omega} q_{w} \psi_{p} \\
\int_{\Omega} p_{c} \psi_{s}=\int_{\Omega} p_{c, e q}\left(S_{w}\right) \psi_{s} & -\int_{\Omega} \tau \partial_{t} S_{w} \psi_{s} .
\end{aligned}
$$

We refer to $[5,4,17]$ for existence and uniqueness results for Problem 1 .

Assumptions The analysis below is carried out under the following assumptions:

(A1) For the initial and boundary data one has $s^{0} \in H^{1}(\Omega), p_{n}^{D}(x) \in H^{\frac{1}{2}}(\Gamma)$ and a function $s^{D}(x) \in H^{\frac{1}{2}}(\Gamma)$ exists s.t. $p_{c}^{D}(x)=p_{c, e q}\left(s^{D}(x)\right)$. Further, the initial and boundary conditions are compatible.

(A2) The permeability matrix $K \in \mathbb{R}^{d \times d}$ is symmetric and positive definite, i.e. there exist two constants $\bar{\kappa}$ and $\underline{\kappa}$, s.t., for any vector $x \in \mathbb{R}^{d}$, the following holds:

$$
\underline{\kappa}\|x\|^{2} \leq x^{T} K x \leq \bar{\kappa}\|x\|^{2}
$$

(A3) The equilibrium capillary pressure function $p_{c, e q}(\cdot)$ is in $C^{2}(\mathbb{R})$, and is assumed positive, bounded and decreasing. Further we assume that there exist $L_{p_{c, e q}}, l_{p_{c, e q}}>0$ such that for all $S \in \mathbb{R}$ it holds

$$
0<l_{p_{c, e q}} \leq p_{c, e q}^{\prime}(\cdot) \leq L_{p_{c, e q}}<\infty .
$$

(A4) The functions $\lambda_{w}(\cdot)$ and $\lambda_{n}(\cdot)$ are Lipschitz-continuous and two constants $\overline{\lambda_{\alpha}}, \underline{\lambda_{\alpha}}>0$ exist such that for all $S \in \mathbb{R}$,

$$
0<\underline{\lambda_{\alpha}}<\lambda_{\alpha}(S)<\overline{\lambda_{\alpha}}<\infty, \quad(\alpha \in\{w, n\}) .
$$

(A5) The dynamic capillary pressure function $\tau(\cdot)$ is in $C^{2}(\mathbb{R})$, positive, bounded, and decreasing. Letting $T(\cdot)$ denote its primitive, we assume that there exist $L_{T}, l_{T}>0$ such that for all $S \in \mathbb{R}$ one has

$$
0<l_{T} \leq \tau(S) \leq L_{T}<\infty
$$




\section{NUMERICAL APPROXIMATION OF TWO-PHASE FLOW IN POROUS MEDIA WITH DYNAMIC CAPILLARITY}

In this section we present the discretization of (5)-(7). The scheme uses the backward Euler time stepping and and dG method for the discretization in space. Also, we introduce the linear, iterative $L$-scheme employed for solving the nonlinear systems appearing at each time step. Before doing so we present some notations that will be used below.

Preliminaries For the spatial discretization we let $\mathcal{T}$ be a decomposition of the domain $\Omega$ into $N$ non-degenerate elements $T_{r}$. We assume that $\mathcal{T}$ is admissible in the sense of the Definition 2.1 in Di Pietro and Ern (2010) [7]. Let $\mathcal{F}$ denote the union of all faces $F_{j}$, and let $h$ be the maximal diameter of the elements.

Given $T_{r} \in \mathcal{T}$ and $F_{r} \in \mathcal{F}$, we define a set $F\left(T_{r}\right)$ of all the faces associated with the element $T_{r}$, s.t.,

$$
F\left(T_{r}\right):=\left\{\bigcup_{F_{j} \in \mathcal{F}} F_{j}: F_{j} \subset T_{r}\right\},
$$

and, a set $T\left(F_{r}\right)$ of all the elements sharing the face $F_{r}$, s.t.,

$$
T\left(F_{r}\right):=\left\{\bigcup_{T_{j} \in \mathcal{T}} T_{j}: F_{r} \subset T_{j}\right\} .
$$

In the conformig case, $T\left(F_{r}\right)$ consists of exactly two elements.

With each face $F \in \mathcal{F}$ connecting element $T_{i}$ and $T_{j}$, we associate a normal-vector $\vec{n}$ directed from $T_{i}$ to $T_{j}(j>i)$.

Given $k \in \mathbb{N}, \Pi^{k}(T)$ denotes the space of polynomials on $T$ with degree $\leq k$. The dG method provides a piecewise polynomial approximation for the saturation and the pressures. Specifically, $S_{w}$ is approximated in the broken Sobolev space with polynomials of order $k_{s}$

$$
V_{h}^{s}(\Omega):=\left\{v \in L^{2}(\Omega):\left.v\right|_{T} \in \Pi^{k_{s}}(T) \text { for all } T \in \mathcal{T}\right\},
$$

whereas for the pressures $p_{n}$ and $p_{c}$ we consider the broken Sobolev space with polynomials of order $k_{p}$

$$
V_{h}^{p}(\Omega):=\left\{v \in L^{2}(\Omega):\left.v\right|_{T} \in \Pi^{k_{p}}(T) \text { for all } T \in \mathcal{T}\right\}
$$

Let $V_{h}(\Omega)$ be any broken Sobolev space of piecewise polynomials of maximal degree $k \in \mathbb{N}$, and $\in V_{h}(\Omega)$. Let $F \in \mathcal{F}$ and $T_{i}, T_{j} \in T(F)$. Then $\psi^{i}, \psi^{j}$ are the traces of $\psi$ on $F$ from the sides of $T_{i}$, respectively $T_{j}: \psi^{i}=\left.\left(\left.\psi\right|_{T^{i}}\right)\right|_{F}, \psi^{j}=\left.\left(\left.\psi\right|_{T^{j}}\right)\right|_{F}$. These are used to we define the jump $\llbracket \rrbracket$ and 
the average $\{\cdot\}$ over the face $F$ as

$$
\begin{array}{ll}
\text { when } F \text { is an interior face : } & \llbracket \psi \rrbracket=\left(\psi^{i}-\psi^{j}\right) \quad \text { and } \quad\{\psi\}=\frac{1}{2}\left(\psi^{i}+\psi^{j}\right), \\
\text { when } F \text { is a boundary face : } & \llbracket \psi \rrbracket=\psi^{i} \quad \text { and } \quad\{\psi\}=\psi^{i} .
\end{array}
$$

where, the interior face connects elements $T^{i}$ and $T^{j}$ with $i<j$, and the boundary face has no element adjacent to $T_{i}$.

For proving the convergence of the scheme we use the following norm on the broken SobolevSpace

$$
\|v\|_{\Omega, D G}^{2}:=\sum_{T_{r} \in \mathcal{T}}\|\nabla v\|_{T_{r}, 0}^{2}+\sum_{F_{r} \in \mathcal{F}} \frac{1}{\left|F_{r}\right|}\|\llbracket v \rrbracket\|_{F_{r}, 0}^{2},
$$

and the lemma below (see e.g. [7]):

\section{Lemma 1}

Let $k \in \mathbb{N}$ and $V_{h}(\Omega)$ the broken Sobolev-Space of piecewise polynomials of maximal degree $k$. Given $q$ such that

$$
\begin{aligned}
& 1 \leq q \leq \frac{2 d}{d-2} \quad \text { if } d \geq 3, \quad \text { or } \\
& 1 \leq q<\infty \quad \text { if } d=2,
\end{aligned}
$$

there exists a constant $\hat{C}$ depending on the maximal polynomial degree $k$, the meshparameters and $|\Omega|$ (the volume of $\Omega$ ) such that for all $v \in V_{h}(\Omega)$ one has

$$
\|v\|_{L^{q}(\Omega)} \leq \hat{C}\|v\|_{\Omega, D G}
$$

Further, the following trace inequalities will be used (see e.g. $[35,33,8]$ )

\section{Lemma 2}

With $\gamma_{0}$ denoting the trace operator, there exists a constant $C_{t}$ independent of the mesh size $h$ such that for any $T \in \mathcal{T}$ with $F \in F(T)$ and for all $v \in H^{k}(T)$, the following holds:

$$
\left\|\gamma_{0} v\right\|_{0, F} \leq C_{t} \sqrt{\frac{1}{|F|}}\left(\|v\|_{0, T}+|F|\|\nabla v\|_{0, T}\right)
$$

Further, a function $f(\cdot)$ exists such that if $v \in \Pi^{k}(T)$ one has

$$
\left\|\gamma_{0} v\right\|_{0, F} \leq C_{t} \sqrt{\frac{f(k)}{|F|}}\|v\|_{0, T}
$$


Also, the following elementary lemma will be used (see [11])

\section{Lemma 3}

Let $\tilde{C}$ be the maximal number of elements sharing one face, and let $A: \mathcal{T} \rightarrow[0, \infty)$ be a function defined on the triangularization $\mathcal{T}$. Then, the following inequality holds:

$$
\sum_{F_{r}} \sum_{T\left(F_{r}\right)} A(T) \leq \tilde{C} \sum_{T_{r}} A\left(T_{r}\right)
$$

Finally, we will use the following elementary (in-)equalities for real numbers. Let $a, b \in \mathbb{R}$ and $\epsilon>0$, then

$$
\begin{aligned}
& (a-b) \cdot a=\frac{1}{2}(a-b)^{2}+\frac{1}{2}\left(a^{2}-b^{2}\right) \\
& a b \leq \frac{\epsilon}{2} a^{2}+\frac{1}{2 \epsilon} b^{2}
\end{aligned}
$$

Discretization in time For the discretization in time, we use an implicit Euler scheme. Let $N \in \mathbb{N}$ and $\Delta t=T / N$. We divide the time domain $[0, T]$ into $N$ uniform intervals and denote the $i$-th discrete time-step by $t_{i}\left(t_{i}=i \cdot \Delta t\right)$.

Given the function $g:[0, T] \times \Omega \rightarrow \mathbb{R}$, its time derivative at $t=t^{n+1}$ is approximated by

$$
\partial^{-} g^{n+1}:=\frac{g\left(t^{n+1}, x\right)-g\left(t^{n}, x\right)}{\Delta t} .
$$

For simplicity we write $g^{n}=g\left(t_{n}, \cdot\right)$.

With this, we obtain a sequence of time-discrete problems $(n=0, \ldots, N-1)$ :

\section{Problem 2 (Time discrete problem)}

Given $s_{w}^{n}, p_{n}^{n}$ and $p_{c}^{n}$, find $s_{w}^{n+1}, p_{n}^{n+1}$ and $p_{c}^{n+1}$, s.t., the following holds:

$$
\begin{aligned}
& \frac{s_{w}^{n+1}-s_{w}^{n}}{\Delta t} \phi+\nabla \cdot\left(\lambda_{n}\left(s_{w}^{n+1}\right) K \nabla p_{n}^{n+1}\right)=0 \\
& \frac{s_{w}^{n+1}-s_{w}^{n}}{\Delta t} \phi+\nabla \cdot\left(\lambda_{w}\left(s_{w}^{n+1}\right) K \nabla\left(p_{n}^{n+1}-p_{c}^{n+1}\right)\right)=0 \\
& p_{c}^{n+1}=p_{c, e q}\left(s_{w}^{n+1}\right)-\frac{T\left(s_{w}^{n+1}\right)-T\left(s_{w}^{n}\right)}{\Delta t}
\end{aligned}
$$

Observe that, at each time step, this results into a nonlinear problem. For solving it we propose an iteration scheme that builds on the ideas in [23, 28, 29, 30, 34, 36] (the "L"-scheme). The idea is to construct a sequence of triplets $\left(s_{w}^{n, i-1}, p_{n}^{n, i-1}, p_{c}^{n, i-1}\right)$ converging as $i \rightarrow \infty$ to the solution $\left(s_{w}^{n+1}, p_{n}^{n+1}, p_{c}^{n+1}\right)$ of Problem 2. Recalling Assumptions (A3) and (A5), we let $L_{s}, L_{s, T}>0$ be 
two positive constants satisfying

$$
L_{s} \geq L_{p_{c, e q}} \quad \text { and } \quad L_{s, T} \geq L_{T}
$$

and define the following linearization scheme:

\section{Problem 3 (Linearization scheme)}

Let $i>0$ and $s_{w}^{n, i-1}, p_{n}^{n, i-1}, p_{c}^{n, i-1}$ be given. Find $s_{w}^{n, i}, p_{n}^{n, i}$, and $p_{c}^{n, i}$ such that

$$
\begin{aligned}
& -\frac{s_{w}^{n, i}-s_{w}^{n}}{\Delta t} \phi+\nabla \cdot\left(\lambda_{n}\left(s_{w}^{n, i-1}\right) K \nabla p_{n}^{n, i}\right)=0 \\
& \frac{s_{w}^{n, i}-s_{w}^{n}}{\Delta t} \phi+\nabla \cdot\left(\lambda_{w}\left(s_{w}^{n, i-1}\right) K \nabla\left(p_{n}^{n, i}-p_{c}^{n, i}\right)\right)=0 \\
& L_{s}\left(s_{w}^{n, i}-s_{w}^{n, i-1}\right)-L_{s, T}\left(\frac{s_{w}^{n, i}-s_{w}^{n, i-1}}{\Delta t}\right)+p_{c}^{n, i}-p_{c, e q}\left(s_{w}^{n, i-1}\right)+\frac{T\left(s_{w}^{n, i-1}\right)-T\left(s_{w}^{n}\right)}{\Delta t}=0
\end{aligned}
$$

\section{Remark 1}

Observe that the first two equations are nothing but the semi-implicit discretization of the corresponding in Problem 2, whereas the third equation includes two additional terms involving the parameters $L_{s}$ and $L_{s, T}$. Formally one can see that if the scheme is convergent, these terms are vanishing and the limit solves the nonlinear time discrete problem. Below we prove that the scheme converges indeed, and that this convergence holds for any initial guess. However, since this is an evolution problem, it is natural to use the solution at the previous time step at starting point, i.e. $s_{w}^{n, 0}=s_{w}^{n}, p_{n}^{n, 0}=p_{n}^{n}$, and $p_{c}^{n, 0}=p_{c}^{n}$.

For the discretization in space, we use a discontinuous Galerkin scheme as presented in [16]. When applied to the time discrete problem 2 obtained at at time step $t^{n+1}$, this results into the following fully-discrete algebraic system

Problem 4 (Discrete problem at $t^{n+1}$ )

Let $P_{n}^{n} \in V_{h}^{p}(\Omega), P_{c}^{n} \in V_{h}^{p}(\Omega)$, and $S_{w}^{n} \in V_{h}^{s}(\Omega)$. Find $P_{n}^{n+1} \in V_{h}^{p}(\Omega), P_{c}^{n+1} \in V_{h}^{p}(\Omega)$, and 
$S_{w}^{n+1} \in V_{h}^{s}(\Omega)$, s.t., for all $\psi_{s} \in V_{h}^{s}(\Omega), \psi_{n} \in V_{h}^{p}(\Omega)$, and $\psi_{w} \in V_{h}^{p}(\Omega)$, the following holds:

$$
\begin{aligned}
\text { PDE-1: } & \sum_{T_{r} \in \mathcal{T}} \int_{T_{r}}-\partial^{-} S_{w}^{n+1} \phi \psi_{n}+\sum_{T_{r} \in \mathcal{T}} \int_{T_{r}} \lambda_{n}\left(S_{w}^{n+1}\right) K \nabla P_{n}^{n+1} \nabla \psi_{n} \\
& -\sum_{F_{r} \in \mathcal{F}} \int_{F_{r}}\left\{\lambda_{n}\left(S_{w}^{n+1}\right) K \nabla P_{n}^{n+1} \cdot \vec{n}\right\} \llbracket \psi_{n} \rrbracket \\
& +\theta \sum_{F_{r} \in \mathcal{F}} \int_{F_{r}} \llbracket P_{n}^{n+1} \rrbracket\left\{\lambda_{n}\left(S_{w}^{n+1}\right) K \nabla \psi_{n}\right\}+\sigma_{n} \sum_{F_{r} \in \mathcal{F}} \int_{F_{r}} \frac{f\left(k_{p}\right)}{\left|F_{r}\right|} \llbracket P_{n}^{n+1} \rrbracket \llbracket \psi_{n} \rrbracket \\
= & \theta \sum_{F_{r} \in \Gamma} \int_{F_{r}} \llbracket p_{n}^{D} \rrbracket\left\{\lambda_{n}\left(s^{D}\right) K \nabla \psi_{n}\right\}+\sigma_{n} \sum_{F_{r} \in \Gamma} \int_{F_{r}} \frac{f\left(k_{p}\right)}{\left|F_{r}\right|} \llbracket p_{n}^{D} \rrbracket \llbracket \psi_{n} \rrbracket
\end{aligned}
$$

$$
\begin{aligned}
\text { PDE-2: } & \sum_{T_{r} \in \mathcal{T}} \int_{T_{r}} \partial^{-} S_{w}^{n+1} \phi \psi_{w}+\sum_{T_{r} \in \mathcal{T}} \int_{T_{r}} \lambda_{w}\left(S_{w}^{n+1}\right) K \nabla\left(P_{n}^{n+1}-P_{c}^{n+1}\right) \nabla \psi_{w} \\
& -\sum_{F_{r} \in \mathcal{F}} \int_{F_{r}}\left\{\lambda_{w}\left(S_{w}^{n+1}\right) K \nabla\left(P_{n}^{n+1}-P_{c}^{n+1}\right) \cdot \vec{n}\right\} \llbracket \psi_{w} \rrbracket \\
& +\theta \sum_{F_{r} \in \mathcal{F}} \int_{F_{r}}\left\{\lambda_{w}\left(S_{w}^{n+1}\right) K \nabla \psi_{w} \cdot \vec{n}\right\} \llbracket P_{n}^{n+1}-P_{c}^{n+1} \rrbracket \\
& +\sigma_{w} \sum_{F_{r} \in \mathcal{F}} \int_{F_{r}} \frac{f\left(k_{p}\right)}{\left|F_{r}\right|} \llbracket P_{n}^{n+1}-P_{c}^{n+1} \rrbracket \llbracket \psi_{w} \rrbracket \\
= & \theta \sum_{F_{r} \in \Gamma} \int_{F_{r}}\left\{\lambda_{w}\left(s^{D}\right) K \nabla \psi_{w} \cdot \vec{n}\right\} \llbracket p_{n}^{D}-p_{c}^{D} \rrbracket \\
+ & \sigma_{w} \sum_{F_{r} \in \Gamma} \int_{F_{r}} \frac{f\left(k_{p}\right)}{\left|F_{r}\right|} \llbracket p_{n}^{D}-p_{c}^{D} \rrbracket \llbracket \psi_{w} \rrbracket
\end{aligned}
$$

$$
\text { ODE-Pc: } \sum_{T_{r} \in \mathcal{T}} \int_{T_{r}} P_{c}^{n+1} \psi_{s}=\sum_{T_{r} \in \mathcal{T}} \int_{T_{r}} p_{c, e q}\left(S_{w}^{n+1}\right) \psi_{s}-\sum_{T_{r} \in \mathcal{T}} \int_{T_{r}} \partial^{-} T\left(S_{w}^{n+1}\right) \psi_{s}
$$

The parameters $\sigma_{n}$ and $\sigma_{w}$ penalize discontinuities in the solutions (i.e., jumps) over the faces. The choice of $\theta=-1$ gives the non-symmetric- (NIPG) scheme, $\theta=0$ gives the incomplete- (IIP) scheme, and $\theta=1$ gives the symmetric-interior-penalty (SIPG) scheme.

Starting from Problem 3 and with the parameters $L_{s}, L_{s, T}$ satisfying (23), the fully discrete linearized scheme becomes

Problem 5 (Fully discrete linearization scheme)

Let $P_{n}^{n} \in V_{h}^{p}(\Omega), P_{c}^{n} \in V_{h}^{p}(\Omega)$, and $S_{w}^{n} \in V_{h}^{s}(\Omega)$. Given $P_{n}^{n, i-1} \in V_{h}^{p}(\Omega), P_{c}^{n, i-1} \in V_{h}^{p}(\Omega)$, and $S_{w}^{n, i-1} \in V_{h}^{s}(\Omega)$ with $P_{n}^{n+1,0}=P_{n}^{n}, P_{c}^{n+1,0}=P_{c}^{n}$, and $S_{w}^{n+1,0}=S_{w}^{n}$, find $P_{n}^{n, i} \in V_{h}^{p}(\Omega), P_{c}^{n, i} \in$ $V_{h}^{p}(\Omega)$, and $S_{w}^{n, i} \in V_{h}^{s}(\Omega)$, s.t., for all $\psi_{s} \in V_{h}^{s}(\Omega), \psi_{n} \in V_{h}^{p}(\Omega)$, and $\psi_{w} \in V_{h}^{p}(\Omega)$, the following 
holds:

$$
\begin{aligned}
\text { PDE-1: } & -\sum_{T_{r} \in \mathcal{T}} \int_{T_{r}} \partial^{-} S_{w}^{n, i} \phi \psi_{n}+\sum_{T_{r} \in \mathcal{T}} \int_{T_{r}} \lambda_{n}\left(S_{w}^{n, i-1}\right) K \nabla P_{n}^{n, i} \nabla \psi_{n} \\
& -\sum_{F_{r} \in \mathcal{F}} \int_{F_{r}}\left\{\lambda_{n}\left(S_{w}^{n, i-1}\right) K \nabla P_{n}^{n, i} \cdot \vec{n}\right\} \llbracket \psi_{n} \rrbracket \\
& +\theta \sum_{F_{r} \in \mathcal{F}} \int_{F_{r}} \llbracket P_{n}^{n, i} \rrbracket\left\{\lambda_{n}\left(S_{w}^{n, i-1}\right) K \nabla \psi_{n}\right\}+\sigma_{n} \sum_{F_{r} \in \mathcal{F}} \int_{F_{r}} \frac{f\left(k_{p}\right)}{\left|F_{r}\right|} \llbracket P_{n}^{n, i} \rrbracket \llbracket \psi_{n} \rrbracket \\
= & \theta \sum_{F_{r} \in \Gamma} \int_{F_{r}} \llbracket p_{n}^{D} \rrbracket\left\{\lambda_{n}\left(s^{D}\right) K \nabla \psi_{n}\right\}+\sigma_{n} \sum_{F_{r} \in \Gamma} \int_{F_{r}} \frac{f\left(k_{p}\right)}{\left|F_{r}\right|} \llbracket p_{n}^{D} \rrbracket \llbracket \psi_{n} \rrbracket
\end{aligned}
$$

PDE-2: $\sum_{T_{r} \in \mathcal{T}} \int_{T_{r}} \partial^{-} S_{w}^{n, i} \phi \psi_{w}+\sum_{T_{r} \in \mathcal{T}} \int_{T_{r}} \lambda_{w}\left(S_{w}^{n, i-1}\right) K \nabla P_{w}^{n, i} \nabla \psi_{w}$

$$
\begin{aligned}
& -\sum_{F_{r} \in \mathcal{F}} \int_{F_{r}}\left\{\lambda_{w}\left(S_{w}^{n, i-1}\right) K \nabla P_{w}^{n, i} \cdot \vec{n}\right\} \llbracket w \rrbracket+\theta \sum_{F_{r} \in \mathcal{F}} \int_{F_{r}}\left\{\lambda_{w}\left(S_{w}^{n, i-1}\right) K \nabla \psi_{w} \cdot \vec{n}\right\} \llbracket P_{w}^{n, i} \rrbracket \\
& +\sigma_{w} \sum_{F_{r} \in \mathcal{F}} \int_{F_{r}} \frac{f\left(k_{p}\right)}{\left|F_{r}\right|} \llbracket P_{w}^{n, i} \rrbracket \llbracket \psi_{w} \rrbracket=\theta \sum_{F_{r} \in \Gamma} \int_{F_{r}}\left\{\lambda_{w}\left(s^{D}\right) K \nabla \psi_{w} \cdot \vec{n}\right\} \llbracket p_{n}^{D}-p_{c}^{D} \rrbracket \\
& +\sigma_{w} \sum_{F_{r} \in \Gamma} \int_{F_{r}} \frac{f\left(k_{p}\right)}{\left|F_{r}\right|} \llbracket p_{n}^{D}-p_{c}^{D} \rrbracket \llbracket \psi_{w} \rrbracket
\end{aligned}
$$

$$
\begin{aligned}
\text { ODE-Pc: } & \sum_{T_{r} \in \mathcal{T}} \int_{T_{r}} L_{s}\left(S_{w}^{n, i}-S_{w}^{n, i-1}\right) \psi_{s}+\sum_{T_{r} \in \mathcal{T}} \int_{T_{r}} L_{s, T}\left(\frac{S_{w}^{n, i}-S_{w}^{n, i-1}}{\Delta t}\right) s \\
& +\sum_{T_{r} \in \mathcal{T}} \int_{T_{r}} P_{c}^{n, i} \psi_{s}-\sum_{T_{r} \in \mathcal{T}} \int_{T_{r}} p_{c, e q}\left(S_{w}^{n, i-1}\right) \psi_{s}+\sum_{T_{r} \in \mathcal{T}} \int_{T_{r}} \frac{T\left(S_{w}^{n, i-1}\right)-T\left(S_{w}^{n}\right)}{\Delta t} \psi_{s}=0
\end{aligned}
$$

In line with Remark 1, the solution at the previous time step is chosen as initial guess for the fully discrete the iteration scheme. However, the convergence result proved in the next section does not require this starting point.

\section{CONVERGENCE ANALYSIS OF THE FULLY DISCRETE LINEARIZATION SCHEME}

Here we give the rigorous proof for the convergence of the linear iterative method introduced as Problem 3, which is then employed for solving the fully discrete (dG/backward Euler) scheme approximating the solution of Problem 1. We refer to [16] for the convergence of this scheme, where the existence of solutions for the fully discrete nonlinear system, as well as apriori error estimates are obtained. In what follows we use the following notations for the errors at the $i$-th iteration:

$$
e_{s}^{i}=S_{w}^{n, i}-S_{w}^{n+1}, \quad e_{p_{\alpha}}^{i}=P_{\alpha}^{n, i}-P_{\alpha}^{n+1},
$$


where $\alpha=n, w, c$. To simplify the writing, we also use the following notation for the errors in $\lambda_{n}(\cdot)$ $\lambda_{w}(\cdot), T(\cdot)$ and $p_{c, e q}(\cdot)$, respectively:

$$
\begin{aligned}
& e_{\lambda_{n}}^{i}=\lambda_{n}\left(S_{w}^{n, i}\right)-\lambda_{n}\left(S_{w}^{n+1}\right), \quad e_{\lambda_{w}}^{i}=\lambda_{w}\left(S_{w}^{n, i}\right)-\lambda_{w}\left(S_{w}^{n+1}\right), \\
& e_{p_{c, e q}}^{i}=p_{c, e q}\left(S_{w}^{n, i}\right)-p_{c, e q}\left(S_{w}^{n+1}\right), \quad e_{T}^{i}=T\left(S_{w}^{n, i}\right)-T\left(S_{w}^{n+1}\right),
\end{aligned}
$$

The following theorem gives the convergence of the iteration. It is proved under a mild restriction on the time step, which is uniform w.r.t. the spatial mesh.

\section{Theorem 1}

\section{Convergence L-scheme}

Under assumptions (A1)-(A5) and if $\Delta t$ is small enough, the iterative scheme (27)(29) converges linearly. 
respectively to get:

\section{PDE-1:}

$-\sum_{T_{r} \in \mathcal{T}} \int_{T_{r}}\left(\partial^{-} S_{w}^{n, i}-\partial^{-} S_{w}^{n+1}\right) \phi \psi_{n}$

$+\sum_{T_{r} \in \mathcal{T}} \int_{T_{r}}\left(\lambda_{n}\left(S_{w}^{n, i-1}\right) K \nabla P_{n}^{n, i}-\lambda_{n}\left(S_{w}^{n+1}\right) K \nabla P_{n}^{n+1}\right) \nabla \psi_{n}$

$-\sum_{F_{r} \in \mathcal{F}} \int_{F_{r}}\left\{\left(\lambda_{n}\left(S_{w}^{n, i-1}\right) K \nabla P_{n}^{n, i}-\lambda_{n}\left(S_{w}^{n+1}\right) K \nabla P_{n}^{n+1}\right) \cdot \vec{n}\right\} \llbracket \psi_{n} \rrbracket$

$+\theta \sum_{F_{r} \in \mathcal{F}} \int_{F_{r}} \llbracket P_{n}^{n, i} \rrbracket\left\{\lambda_{n}\left(S_{w}^{n, i-1}\right) K \nabla \psi_{n}\right\}-\llbracket P_{n}^{n+1} \rrbracket\left\{\lambda_{n}\left(S_{w}^{n+1}\right) K \nabla \psi_{n}\right\}$

$+\sigma_{n} \sum_{F_{r} \in \mathcal{F}} \int_{F_{r}} \frac{f\left(k_{p}\right)}{\left|F_{r}\right|} \llbracket P_{n}^{n, i}-P_{n}^{n+1} \rrbracket \llbracket \psi_{n} \rrbracket=0$

\section{PDE-2:}

$$
\begin{aligned}
& \sum_{T_{r} \in \mathcal{T}} \int_{T_{r}}\left(\partial^{-} S_{w}^{n, i}-\partial^{-} S_{w}^{n+1}\right) \phi \psi_{w} \\
+ & \sum_{T_{r} \in \mathcal{T}} \int_{T_{r}}\left(\lambda_{w}\left(S_{w}^{n, i-1}\right) K \nabla\left(P_{n}^{n, i}-P_{c}^{n, i}\right)-\lambda_{w}\left(S_{w}^{n+1}\right) K \nabla\left(P_{n}^{n+1}-P_{c}^{n+1}\right) \nabla \psi_{w}\right. \\
- & \sum_{F_{r} \in \mathcal{F}} \int_{F_{r}}\left\{\left(\lambda_{w}\left(S_{w}^{n, i-1}\right) K \nabla\left(P_{n}^{n, i}-P_{c}^{n, i}\right)-\lambda_{w}\left(S_{w}^{n+1}\right) K \nabla\left(P_{n}^{n+1}-P_{c}^{n+1}\right)\right) \cdot \vec{n}\right\} \llbracket \psi_{w} \rrbracket \\
+ & \theta \sum_{F_{r} \in \mathcal{F}} \int_{F_{r}}\left\{\lambda_{w}\left(S_{w}^{n, i-1}\right) K \nabla \psi_{w} \cdot \vec{n}\right\} \llbracket P_{n}^{n, i}-P_{c}^{n, i} \rrbracket-\left\{\lambda_{w}\left(S_{w}^{n+1}\right) K \nabla \psi_{w} \cdot \vec{n}\right\} \llbracket P_{n}^{n+1}-P_{c}^{n+1} \rrbracket \\
+ & \sigma_{w} \sum_{F_{r} \in \mathcal{F}} \int_{F_{r}} \frac{f\left(k_{p}\right)}{\left|F_{r}\right|} \llbracket\left(P_{n}^{n, i}-P_{c}^{n, i}\right)-\left(P_{n}^{n+1}-P_{c}^{n+1}\right) \rrbracket \llbracket \psi_{w} \rrbracket=0
\end{aligned}
$$

\section{ODE-Pc:}

$$
\begin{aligned}
& \sum_{T_{r} \in \mathcal{T}} \int_{T_{r}} L_{s}\left(S_{w}^{n, i}-S_{w}^{n, i-1}\right) \psi_{s}+\sum_{T_{r} \in \mathcal{T}} \int_{T_{r}} L_{s, T}\left(\frac{S_{w}^{n, i}-S_{w}^{n, i-1}}{\Delta t}\right) s \\
+ & \sum_{T_{r} \in \mathcal{T}} \int_{T_{r}}\left(P_{c}^{n, i}-P_{c}^{n+1}\right) \psi_{s}-\sum_{T_{r} \in \mathcal{T}} \int_{T_{r}}\left(p_{c, e q}\left(S_{w}^{n, i-1}\right)-p_{c, e q}\left(S_{w}^{n+1}\right)\right) \psi_{s} \\
+ & \sum_{T_{r} \in \mathcal{T}} \int_{T_{r}}\left(\partial^{-} T\left(S_{w}^{n, i-1}\right)-\partial^{-} T\left(S_{w}^{n+1}\right)\right) \psi_{s}=0
\end{aligned}
$$

We proceed by obtaining error estimates separately for the phase pressures and for the capillary pressure. These estimates are then used to prove the convergence of the iteration. 
Estimate for the non-wetting phase Taking $\psi_{n}=e_{p_{n}}^{i}$ in (32) gives

$$
\begin{aligned}
& -\sum_{T_{r} \in \mathcal{T}} \int_{T_{r}} \partial^{-} e_{s}^{i} \phi e_{p_{n}}^{i}+\sum_{T_{r} \in \mathcal{T}} \int_{T_{r}} \lambda_{n}\left(S_{w}^{n, i-1}\right) K\left|\nabla e_{p_{n}}^{i}\right|^{2}+\sigma_{n} \sum_{F_{r} \in \mathcal{F}} \int_{F_{r}} \frac{f\left(k_{p}\right)}{\left|F_{r}\right|} \llbracket e_{p_{n}}^{i} \rrbracket^{2} \\
= & -\sum_{T_{r} \in \mathcal{T}} \int_{T_{r}}\left(\lambda_{n}\left(S_{w}^{n, i-1}\right)-\lambda_{n}\left(S_{w}^{n+1}\right)\right) K \nabla P_{n}^{n+1} \nabla e_{p_{n}}^{i} \\
& +(1-\theta) \sum_{F_{r} \in \mathcal{F}} \int_{F_{r}} \llbracket e_{p_{n}}^{i} \rrbracket\left\{\lambda_{n}\left(S_{w}^{n, i-1}\right) K \nabla e_{p_{n}}^{i}\right\} \\
& \left.-\sum_{F_{r} \in \mathcal{F}} \int_{F_{r}}\left\{\left(\lambda_{n}\left(S_{w}^{n, i-1}\right)-\lambda_{n}\left(S_{w}^{n+1}\right)\right) K \nabla P_{n}^{n+1}\right) \cdot \vec{n}\right\} \llbracket e_{p_{n}}^{i} \rrbracket \\
& -\sum_{F_{r} \in \mathcal{F}} \int_{F_{r}} \theta\left\{\left(\lambda_{n}\left(S_{w}^{n, i-1}\right)-\lambda_{n}\left(S_{w}^{n+1}\right)\right) K \nabla e_{p_{n}}^{i}\right\} \llbracket P_{n}^{n+1} \rrbracket \\
= & : P_{1}+P_{2}+P_{3}+P_{4}
\end{aligned}
$$

For $P_{4}$ one gets

$$
\left|P_{4}\right| \leq\left\|P_{n}^{n+1}\right\|_{\Omega, \infty} \theta \sum_{F_{r} \in \mathcal{F}} \int_{F_{r}}\left|\left\{\left(\lambda_{n}\left(S_{w}^{n, i-1}\right)-\lambda_{n}\left(S_{w}^{n+1}\right)\right) K \nabla e_{p_{n}}^{i} \cdot \vec{n}\right\}\right| .
$$

Using (19), Lemma 3, the Cauchy-Schwarz inequality and (21) leads to

$$
\begin{aligned}
\left|P_{4}\right| & \leq\left\|P_{n}^{n+1}\right\|_{\Omega, \infty} \theta \sum_{T_{r} \in \mathcal{T}} \frac{1}{\sqrt{\left|F_{r}\right|}} \tilde{C} C_{t} \sqrt{\frac{f(k)}{\left|F_{r}\right|}}\left\|e_{\lambda_{n}}^{i-1}\right\|_{T_{r}, 0} \tilde{C} C_{t} \sqrt{\frac{f(k)}{\left|F_{r}\right|}} \sqrt{\left|F_{r}\right|}\left\|K^{\frac{1}{2}} \nabla e_{p_{n}}^{i}\right\|_{T_{r}, 0} \\
& \leq \sqrt{\sum_{T_{r} \in \mathcal{T}}\left\|P_{n}^{n+1}\right\|_{\Omega, \infty}^{2} \theta^{2} \tilde{C}^{4} C_{t}^{4} \frac{f^{2}(k)}{\left|F_{r}\right|^{2}}\left\|e_{\lambda_{n}}^{i-1}\right\|_{T_{r}, 0}^{2}} \sqrt{\sum_{T_{r} \in \mathcal{T}}\left\|K^{\frac{1}{2}} \nabla e_{p_{n}}^{i}\right\|_{T_{r}, 0}^{2}} \\
& \leq \frac{1}{2 \epsilon_{4}}\left\|P_{n}^{n+1}\right\|_{\Omega, \infty}^{2} \theta^{2} \tilde{C}^{4} C_{t}^{4} \frac{f^{2}(k)}{\left|F_{r}\right|^{2}} \sum_{T_{r} \in \mathcal{T}}\left\|e_{\lambda_{n}}^{i-1}\right\|_{T_{r}, 0}^{2}+\frac{\epsilon_{4}}{2} \sum_{T_{r} \in \mathcal{T}}\left\|K^{\frac{1}{2}} \nabla e_{p_{n}}^{i}\right\|_{T_{r}, 0}^{2}
\end{aligned}
$$

for any $\epsilon_{4}>0$.

For $P_{1}, P_{2}$, and $P_{3}$, after carrying out steps that are similar to the ones in [16]) one obtains for any $\epsilon_{1}, \epsilon_{2}, \epsilon_{3}>0$

$$
\begin{aligned}
& \left|P_{1}\right| \leq \frac{\epsilon_{1}}{2} \sum_{T_{r} \in \mathcal{T}}\left\|K^{\frac{1}{2}} \nabla e_{p_{n}}^{i}\right\|_{T_{r}, 0}^{2}+\frac{1}{2 \epsilon_{1}} C\left\|\nabla P_{n}^{n+1}\right\|_{\Omega, \infty} \sum_{T_{r} \in \mathcal{T}}\left\|e_{\lambda_{n}}^{i-1}\right\|_{T_{r}, 0}^{2} \\
& \left|P_{2}\right| \leq \frac{\epsilon_{2}}{2} \sum_{T_{r} \in \mathcal{T}}\left\|K^{\frac{1}{2}} \nabla e_{p_{n}}^{n+1}\right\|_{T_{r}, 0}^{2}+(1-\theta)^{2} \frac{1}{2 \epsilon_{2}} \bar{\lambda}_{n}^{2} C_{t}^{2} \tilde{C}^{2} \sum_{F_{r}} \frac{f\left(k_{p}\right)}{\left|F_{r}\right|}\left\|\llbracket e_{p_{n}}^{n+1} \rrbracket\right\|_{F_{r}, 0}^{2} \\
& \left|P_{3}\right| \leq \frac{\epsilon_{3}}{2} \sum_{F_{r} \in \mathcal{F}} \frac{f\left(k_{p}\right)}{\left|F_{r}\right|}\left\|\llbracket e_{p_{n}}^{i} \rrbracket\right\|_{F_{r}, 0}^{2}+\frac{1}{2 \epsilon_{3}}\left\|K \nabla P_{n}^{n+1}\right\|_{\Omega, \infty} \tilde{C}^{2} C_{t}^{2} \sum_{T_{r} \in \mathcal{T}}\left\|e_{\lambda_{n}}^{i-1}\right\|_{T_{r}, 0}^{2} .
\end{aligned}
$$

Observe that the estimate for $P_{3}$ involves the essential boundedness for the gradient of the pressure $P_{n}^{n+1}$. Assumptions (A2)-(A5) ensure that the problem remains nondegenerate and therefore the pressures have essential bounded gradients (see e.g. [5]). These estimates can be extended to the time 
discrete problems, with the time derivative of the saturation being replaced by the finite difference approximation, noting that these divided differences satisfy the same bounds as $\partial_{t} s$ (see [4]). The extension to the finite element approximation follows from [25] (also see [22]).

Combining the estimates for $\left|P_{1}\right|$ to $\left|P_{4}\right|$ and choosing $\epsilon_{3}=\sigma_{n}$ and $\epsilon_{1}=\epsilon_{2}=\epsilon_{4}=\frac{\lambda_{n}}{3}$ gives the following estimate for the non-wetting phase:

$$
\begin{aligned}
& -\sum_{T_{r} \in \mathcal{T}} \int_{T_{r}}\left(\partial^{-} e_{s}^{i}\right) \phi e_{p_{n}}^{i}+\frac{\lambda_{n}}{2} \sum_{T_{r} \in \mathcal{T}}\left\|K^{\frac{1}{2}} \nabla e_{p_{n}}^{i}\right\|_{T_{r}, 0}^{2} \\
& +\left(\frac{\sigma_{n}}{2}-(1-\theta)^{2} \frac{3{\overline{\lambda_{n}}}^{2} C_{t}^{2} \tilde{C}^{2}}{2 \underline{\lambda_{n}}}\right) \sum_{F_{r} \in \mathcal{F}} \frac{f\left(k_{p}\right)}{\left|F_{r}\right|}\left\|\llbracket e_{p_{n}}^{i} \rrbracket\right\|_{F_{r}, 0}^{2} \\
& \leq\left(C_{n, 1}+C_{n, 2} \frac{\theta^{2}}{\left|F_{r}\right|^{2}}\right) \sum_{T_{r} \in \mathcal{T}}\left\|e_{\lambda_{n}}^{i-1}\right\|_{T_{r}, 0}^{2},
\end{aligned}
$$

for some $C_{n, 1}, C_{n, 2}$ not depending on the discretization parameters.

Estimate for the wetting phase As for the nonwetting phase, taking $\psi_{w}=e_{p_{w}}^{i}$ into (33) gives

$$
\begin{aligned}
& \sum_{T_{r} \in \mathcal{T}} \int_{T_{r}}\left(\partial^{-} e_{s}^{i}\right) \phi e_{p_{w}}^{i}+\frac{\lambda_{w}}{2} \sum_{T_{r} \in \mathcal{T}}\left\|K^{\frac{1}{2}} \nabla e_{p_{w}}^{i}\right\|_{T_{r}, 0}^{2} \\
& +\left(\frac{\sigma_{w}}{2}-(1-\theta)^{2} \frac{3{\overline{\lambda_{w}}}^{2} C_{t}^{2} \tilde{C}^{2}}{2 \underline{\lambda_{w}}}\right) \sum_{F_{r} \in \mathcal{F}} \frac{f\left(k_{p}\right)}{\left|F_{r}\right|}\left\|\llbracket e_{p_{w}}^{i}\right\| \|_{F_{r}, 0}^{2} \\
& \leq\left(C_{w, 1}+C_{w, 2} \frac{\theta^{2}}{\left|F_{r}\right|^{2}}\right) \sum_{T_{r} \in \mathcal{T}}\left\|e_{\lambda_{w}}^{i-1}\right\|_{T_{r}, 0}^{2} .
\end{aligned}
$$

for some $C_{w, 1}, C_{w, 2}$ not depending on the discretization parameters.

Estimate for the capillary pressure With $\psi_{s}=e_{s}^{i}$ in (34) one obtains

$$
\begin{aligned}
& \sum_{T_{r} \in \mathcal{T}} \int_{T_{r}} L_{s}\left(S_{w}^{n, i}-S_{w}^{n, i-1}\right) e_{s}^{i}+\sum_{T_{r} \in \mathcal{T}} \int_{T_{r}} L_{s, T}\left(\frac{S_{w}^{n, i}-S_{w}^{n, i-1}}{\Delta t}\right) e_{s}^{i}+\sum_{T_{r} \in \mathcal{T}} \int_{T_{r}} e_{p_{c}}^{i} e_{s}^{i} \\
& -\sum_{T_{r} \in \mathcal{T}} \int_{T_{r}}\left(p_{c, e q}\left(S_{w}^{n, i-1}\right)-p_{c, e q}\left(S_{w}^{n+1}\right)\right) e_{s}^{i}+\sum_{T_{r} \in \mathcal{T}} \int_{T_{r}}\left(\partial^{-} T\left(S_{w}^{n, i-1}\right)-\partial^{-} T\left(S_{w}^{n+1}\right)\right) e_{s}^{i}=0
\end{aligned}
$$

Recalling the notations in (30)-(31) and observing that

$$
\partial^{-} T\left(S_{w}^{n, i-1}\right)-\partial^{-} T\left(S_{w}^{n+1}\right)=\frac{1}{\Delta t}\left(T\left(S_{w}^{n, i-1}\right)-T\left(S_{w}^{n+1}\right)\right),
$$


using (20) in (37) yields

$$
\begin{aligned}
& \frac{L_{s}}{2} \sum_{T_{r} \in \mathcal{T}}\left\|e_{s}^{i}\right\|_{T_{r}, 0}^{2}+\frac{L_{s, T}}{2} \Delta t \sum_{T_{r} \in \mathcal{T}}\left\|\partial^{-} e_{s}^{i}\right\|_{T_{r}, 0}^{2}+\sum_{T_{r} \in \mathcal{T}} \int_{T_{r}} e_{p_{c}}^{i} e_{s}^{i}+\frac{L_{s}}{2} \sum_{T_{r} \in \mathcal{T}}\left\|e_{s}^{i}-e_{s}^{i-1}\right\|_{T_{r}, 0}^{2} \\
+ & \frac{L_{s, T}}{2} \Delta t \sum_{T_{r} \in \mathcal{T}}\left\|\partial^{-} e_{s}^{i}-\partial^{-} e_{s}^{i-1}\right\|_{T_{r}, 0}^{2}-\sum_{T_{r} \in \mathcal{T}} \int_{T_{r}} e_{p_{c, e q}}^{i-1} e_{s}^{i}+\sum_{T_{r} \in \mathcal{T}} \int_{T_{r}} \frac{1}{\Delta t} e_{T}^{i-1} e_{s}^{i} \\
= & \frac{L_{s}}{2} \sum_{T_{r} \in \mathcal{T}}\left\|e_{s}^{i-1}\right\|_{T_{r}, 0}^{2}+\frac{L_{s, T}}{2} \Delta t \sum_{T_{r} \in \mathcal{T}}\left\|\partial^{-} e_{s}^{i-1}\right\|_{T_{r}, 0}^{2} .
\end{aligned}
$$

Since $p_{c, e q}(\cdot)$ is monotone one has

$$
-\left(p_{c, e q}(x)-p_{c, e q}(y)\right)(x-y)=\left|p_{c, e q}(x)-p_{c, e q}(y)\right| \cdot|x-y|,
$$

and similarly for $T(\cdot)$. In this way, (38) becomes

$$
\begin{aligned}
& \frac{L_{s}}{2} \sum_{T_{r} \in \mathcal{T}}\left\|e_{s}^{i}\right\|_{T_{r}, 0}^{2}+\frac{L_{s, T}}{2} \Delta t \sum_{T_{r} \in \mathcal{T}}\left\|\partial^{-} e_{s}^{i}\right\|_{T_{r}, 0}^{2}+\sum_{T_{r} \in \mathcal{T}} \int_{T_{r}} e_{p_{c}}^{i} e_{s}^{i}+\frac{L_{s}}{2} \sum_{T_{r} \in \mathcal{T}}\left\|e_{s}^{i}-e_{s}^{i-1}\right\|_{T_{r}, 0}^{2} \\
+ & \frac{L_{s, T}}{2} \Delta t \sum_{T_{r} \in \mathcal{T}}\left\|\partial^{-} e_{s}^{i}-\partial^{-} e_{s}^{i-1}\right\|_{T_{r}, 0}^{2}+\sum_{T_{r} \in \mathcal{T}} \int_{T_{r}}\left|e_{p_{c, e q}}^{i-1}\right| \cdot\left|e_{s}^{i-1}\right|+\sum_{T_{r} \in \mathcal{T}} \int_{T_{r}}\left|\frac{1}{\Delta t} e_{T}^{i-1}\right| \cdot\left|e_{s}^{i-1}\right| \\
= & \frac{L_{s}}{2} \sum_{T_{r} \in \mathcal{T}}\left\|e_{s}^{i-1}\right\|_{T_{r}, 0}^{2}+\frac{L_{s, T}}{2} \Delta t \sum_{T_{r} \in \mathcal{T}}\left\|\partial^{-} e_{s}^{i-1}\right\|_{T_{r}, 0}^{2} \\
+ & \sum_{T_{r} \in \mathcal{T}} \int_{T_{r}} e_{p_{c, e q}}^{i-1}\left(e_{s}^{i}-e_{s}^{i-1}\right)+\sum_{T_{r} \in \mathcal{T}} \int_{T_{r}} \frac{1}{\Delta t} e_{T}^{i-1}\left(e_{s}^{i-1}-e_{s}^{i}\right) .
\end{aligned}
$$

Using the Lipschitz continuity of $p_{c, e q}(\cdot)$ and $T(\cdot)$, the Young's inequality gives

$$
\begin{aligned}
& \frac{L_{s}}{2} \sum_{T_{r} \in \mathcal{T}}\left\|e_{s}^{i}\right\|_{T_{r}, 0}^{2}+\frac{L_{s, T}}{2} \Delta t \sum_{T_{r} \in \mathcal{T}}\left\|\partial^{-} e_{s}^{i}\right\|_{T_{r}, 0}^{2}+\sum_{T_{r} \in \mathcal{T}} \int_{T_{r}} e_{p_{c}}^{i} e_{s}^{i}+\frac{L_{s}}{2} \sum_{T_{r} \in \mathcal{T}}\left\|e_{s}^{i}-e_{s}^{i-1}\right\|_{T_{r}, 0}^{2} \\
+ & \frac{L_{s, T}}{2} \Delta t \sum_{T_{r} \in \mathcal{T}}\left\|\partial^{-} e_{s}^{i}-\partial^{-} e_{s}^{i-1}\right\|_{T_{r}, 0}^{2}+\frac{1}{L_{P_{c}}} \sum_{T_{r} \in \mathcal{T}}\left\|e_{p_{c, e q}}^{i-1}\right\|_{T_{r}, 0}^{2}+\frac{1}{L_{T} \Delta t} \sum_{T_{r} \in \mathcal{T}}\left\|e_{T}^{i-1}\right\|_{T_{r}, 0}^{2} \\
\leq & \frac{L_{s}}{2} \sum_{T_{r} \in \mathcal{T}}\left\|e_{s}^{i-1}\right\|_{T_{r}, 0}^{2}+\frac{L_{s, T}}{2} \Delta t \sum_{T_{r} \in \mathcal{T}}\left\|\partial^{-} e_{s}^{i-1}\right\|_{T_{r}, 0}^{2}+\frac{1}{2 L_{s}} \sum_{T_{r} \in \mathcal{T}}\left\|e_{p_{c, e q}}^{i-1}\right\|_{T_{r}, 0}^{2} \\
+ & \frac{L_{s}}{2} \sum_{T_{r} \in \mathcal{T}}\left\|e_{s}^{i}-e_{s}^{i-1}\right\|_{T_{r}, 0}^{2}+\frac{1}{2 L_{s, T} \Delta t} \sum_{T_{r} \in \mathcal{T}}\left\|e_{T}^{i-1}\right\|_{T_{r}, 0}^{2}+\frac{L_{s, T} \Delta t}{2} \sum_{T_{r} \in \mathcal{T}}\left\|\frac{1}{\Delta t}\left(e_{s}^{i-1}-e_{s}^{i}\right)\right\|_{T_{r}, 0}^{2} .
\end{aligned}
$$

Using by (23) we get the following estimate for the capillary pressure

$$
\begin{aligned}
& \frac{L_{s}}{2} \sum_{T_{r} \in \mathcal{T}}\left\|e_{s}^{i}\right\|_{T_{r}, 0}^{2}+\frac{L_{s, T}}{2} \Delta t \sum_{T_{r} \in \mathcal{T}}\left\|\partial^{-} e_{s}^{i}\right\|_{T_{r}, 0}^{2}+\sum_{T_{r} \in \mathcal{T}} \int_{T_{r}} e_{p_{c}}^{i} e_{s}^{i} \\
+ & \frac{1}{2 L_{s}} \sum_{T_{r} \in \mathcal{T}}\left\|e_{p_{c, e q}}^{i-1}\right\|_{T_{r}, 0}^{2}+\frac{1}{2 L_{s, T} \Delta t} \sum_{T_{r} \in \mathcal{T}}\left\|e_{T}^{i-1}\right\|_{T_{r}, 0}^{2} \\
\leq & \frac{L_{s}}{2} \sum_{T_{r} \in \mathcal{T}}\left\|e_{s}^{i-1}\right\|_{T_{r}, 0}^{2}+\frac{L_{s, T}}{2} \Delta t \sum_{T_{r} \in \mathcal{T}}\left\|\partial^{-} e_{s}^{i-1}\right\|_{T_{r}, 0}^{2} .
\end{aligned}
$$


Combined estimate Multiplying (39) by $\frac{\phi}{\Delta t}$ and adding the resulting to the sum of (35) and (36) and observing that

$$
\sum_{T_{r} \in \mathcal{T}} \int_{T_{r}} \frac{\phi}{\Delta t} e_{p_{c}}^{i} e_{s}^{i}+\sum_{T_{r} \in \mathcal{T}} \int_{T_{r}} \partial^{-} e_{s}^{i}\left(\phi e_{p_{w}}^{i}-\phi e_{p_{n}}^{i}\right)=\sum_{T_{r} \in \mathcal{T}} \int_{T_{r}} \frac{\phi}{\Delta t} e_{p_{c}}^{i} e_{s}^{i}-\sum_{T_{r} \in \mathcal{T}} \int_{T_{r}} \partial^{-} e_{s}^{i} e_{p_{c}}^{i}=0
$$

leads to

$$
\begin{aligned}
& \frac{L_{s}}{2} \frac{\phi}{\Delta t} \sum_{T_{r} \in \mathcal{T}}\left\|e_{s}^{i}\right\|_{T_{r}, 0}^{2}+\frac{L_{s, T} \phi}{2} \sum_{T_{r} \in \mathcal{T}}\left\|\partial^{-} e_{s}^{i}\right\|_{T_{r}, 0}^{2} \\
+ & \frac{\phi}{2 L_{s} \Delta t} \sum_{T_{r} \in \mathcal{T}}\left\|e_{p_{c, e q}}^{i-1}\right\|_{T_{r}, 0}^{2}+\frac{\phi}{2 L_{s, T} \Delta t^{2}} \sum_{T_{r} \in \mathcal{T}}\left\|e_{T}^{i-1}\right\|_{T_{r}, 0}^{2} \\
+ & \frac{\lambda_{n}}{2} \sum_{T_{r} \in \mathcal{T}}\left\|K^{\frac{1}{2}} \nabla e_{p_{n}}^{i}\right\|_{T_{r}, 0}^{2}+\left(\frac{\sigma_{n}}{2}-(1-\theta)^{2} \frac{3{\overline{\lambda_{n}}}^{2} C_{t}^{2} \tilde{C}^{2}}{2 \frac{\lambda_{n}}{2}}\right) \sum_{F_{r} \in \mathcal{F}} \frac{f\left(k_{p}\right)}{\left|F_{r}\right|}\left\|\llbracket e_{p_{n}}^{i} \rrbracket\right\|_{F_{r}, 0}^{2} \\
+ & \frac{\lambda_{w}}{2} \sum_{T_{r} \in \mathcal{T}}\left\|K^{\frac{1}{2}} \nabla e_{p_{w}}^{i}\right\|_{T_{r}, 0}^{2}+\left(\frac{\sigma_{w}}{2}-(1-\theta)^{2} \frac{3{\overline{\lambda_{w}}}^{2} C_{t}^{2} \tilde{C}^{2}}{2 \underline{\lambda_{w}}}\right) \sum_{F_{r} \in \mathcal{F}} \frac{f\left(k_{p}\right)}{\left|F_{r}\right|}\left\|\llbracket e_{p_{w}}^{i} \rrbracket\right\|_{F_{r}, 0}^{2} \\
\leq & \left(C_{n, 0}+C_{n, 1}+\frac{C_{n, 2} \theta^{2}}{\left|F_{r}\right|^{2}}\right) \sum_{T_{r} \in \mathcal{T}}\left\|e_{\lambda_{n}}^{i-1}\right\|_{T_{r}, 0}^{2}+\left(C_{w, 0}+C_{w, 1}+\frac{C_{w, 2} \theta^{2}}{\left|F_{r}\right|^{2}}\right) \sum_{T_{r} \in \mathcal{T}}\left\|e_{\lambda_{w}}^{i-1}\right\|_{T_{r}, 0}^{2} \\
+ & \frac{L_{s} \phi}{2 \Delta t} \sum_{T_{r} \in \mathcal{T}}\left\|e_{s}^{i-1}\right\|_{T_{r}, 0}^{2}+\frac{L_{s, T} \phi}{2} \sum_{T_{r} \in \mathcal{T}}\left\|\partial^{-} e_{s}^{i-1}\right\|_{T_{r}, 0}^{2} .
\end{aligned}
$$

After multiplying with $\Delta t$ and rearranging the terms this becomes

$$
\begin{aligned}
& \frac{L_{s} \phi}{2} \sum_{T_{r} \in \mathcal{T}}\left\|e_{s}^{i}\right\|_{T_{r}, 0}^{2}+\frac{\Delta t L_{s, T} \phi}{2} \sum_{T_{r} \in \mathcal{T}}\left\|\partial^{-} e_{s}^{i}\right\|_{T_{r}, 0}^{2} \\
+ & \frac{\phi}{2 L_{s}} \sum_{T_{r} \in \mathcal{T}}\left\|e_{p_{c, e q}}^{i-1}\right\|_{T_{r}, 0}^{2}+\frac{\phi}{2 L_{s, T} \Delta t} \sum_{T_{r} \in \mathcal{T}}\left\|e_{T}^{i-1}\right\|_{T_{r}, 0}^{2} \\
+ & \frac{\Delta t \frac{\lambda_{n}}{2}}{2} \sum_{T_{r} \in \mathcal{T}}\left\|K^{\frac{1}{2}} \nabla e_{p_{n}}^{i}\right\|_{T_{r}, 0}^{2}+\Delta t\left(\frac{\sigma_{n}}{2}-(1-\theta)^{2} \frac{3{\overline{\lambda_{n}}}^{2} C_{t}^{2} \tilde{C}^{2}}{2}\right) \sum_{F_{r} \in \mathcal{F}} \frac{f\left(k_{p}\right)}{\left|F_{r}\right|}\left\|\llbracket e_{p_{n}}^{i}\right\| \|_{F_{r}, 0}^{2} \\
+ & \frac{\Delta t \underline{\lambda_{w}}}{2} \sum_{T_{r} \in \mathcal{T}}\left\|K^{\frac{1}{2}} \nabla e_{p_{w}}^{i}\right\|_{T_{r}, 0}^{2}+\Delta t\left(\frac{\sigma_{w}}{2}-(1-\theta)^{2} \frac{3{\overline{\lambda_{w}}}^{2} C_{t}^{2} \tilde{C}^{2}}{2}\right) \sum_{F_{r} \in \mathcal{F}} \frac{f\left(k_{p}\right)}{\left|F_{r}\right|}\left\|\llbracket e_{p_{w}}^{i}\right\| \|_{F_{r}, 0}^{2} \\
\leq & \Delta t\left(C_{n, 0}+C_{n, 1}+\frac{C_{n, 2} \theta^{2}}{\left|F_{r}\right|^{2}}\right) \sum_{T_{r} \in \mathcal{T}}\left\|e_{\lambda_{n}}^{i-1}\right\|_{T_{r}, 0}^{2}+\Delta t\left(C_{w, 0}+C_{w, 1}+\frac{C_{w, 2} \theta^{2}}{\left|F_{r}\right|^{2}}\right) \sum_{T_{r} \in \mathcal{T}}\left\|e_{\lambda_{w}}^{i-1}\right\|_{T_{r}, 0}^{2} \\
+ & \frac{L_{s} \phi}{2} \sum_{T_{r} \in \mathcal{T}}\left\|e_{s}^{i-1}\right\|_{T_{r}, 0}^{2}+\frac{\Delta t L_{s, T} \phi}{2} \sum_{T_{r} \in \mathcal{T}}\left\|\partial^{-} e_{s}^{i-1}\right\|_{T_{r}, 0}^{2} .
\end{aligned}
$$


Using the Lipschitz continuity of $\lambda_{n}, \lambda_{w}, T^{-1}$, and $p_{c, e q}^{-1}$, this rewrites

$$
\begin{aligned}
& \frac{L_{s} \phi}{2} \sum_{T_{r} \in \mathcal{T}}\left\|e_{s}^{i}\right\|_{T_{r}, 0}^{2}+\frac{\Delta t L_{s, T} \phi}{2} \sum_{T_{r} \in \mathcal{T}}\left\|\partial^{-} e_{s}^{i}\right\|_{T_{r}, 0}^{2} \\
+ & \frac{l_{p_{c, e q}}^{2} \phi}{2 L_{s}} \sum_{T_{r} \in \mathcal{T}}\left\|e_{s}^{i-1}\right\|_{T_{r}, 0}^{2}+\frac{l_{T}^{2} \Delta t \phi}{2 L_{s, T}} \sum_{T_{r} \in \mathcal{T}}\left\|\partial^{-} e_{s}^{i-1}\right\|_{T_{r}, 0}^{2} \\
+ & \frac{\Delta t \underline{\lambda_{n}}}{2} \sum_{T_{r} \in \mathcal{T}}\left\|K^{\frac{1}{2}} \nabla e_{p_{n}}^{i}\right\|_{T_{r}, 0}^{2}+\Delta t\left(\frac{\sigma_{n}}{2}-(1-\theta)^{2} \frac{3{\overline{\lambda_{n}}}^{2} C_{t}^{2} \tilde{C}^{2}}{2 \underline{\lambda_{n}}}\right) \sum_{F_{r} \in \mathcal{F}} \frac{f\left(k_{p}\right)}{\left|F_{r}\right|}\left\|\llbracket e_{p_{n}}^{i} \rrbracket\right\|_{F_{r}, 0}^{2} \\
+ & \frac{\Delta t \underline{\lambda_{w}}}{2} \sum_{T_{r} \in \mathcal{T}}\left\|K^{\frac{1}{2}} \nabla e_{p_{w}}^{i}\right\|_{T_{r}, 0}^{2}+\Delta t\left(\frac{\sigma_{w}}{2}-(1-\theta)^{2} \frac{3{\overline{\lambda_{w}}}^{2} C_{t}^{2} \tilde{C}^{2}}{2 \underline{\lambda_{w}}}\right) \sum_{F_{r} \in \mathcal{F}} \frac{f\left(k_{p}\right)}{\left|F_{r}\right|}\left\|\llbracket e_{p_{w}}^{i} \rrbracket\right\|_{F_{r}, 0}^{2} \\
\leq & \frac{L_{s} \phi}{2} \sum_{T_{r} \in \mathcal{T}}\left\|e_{s}^{i-1}\right\|_{T_{r}, 0}^{2}+\frac{\Delta t L_{s, T} \phi}{2} \sum_{T_{r} \in \mathcal{T}}\left\|\partial^{-} e_{s}^{i-1}\right\|_{T_{r}, 0}^{2} \\
+ & \Delta t\left(L_{\lambda_{n}}^{2} C_{n}+L_{\lambda_{w}}^{2} C_{w}\right)\left(2+\frac{\theta^{2}}{\left|F_{r}\right|^{2}}\right) \sum_{T_{r} \in \mathcal{T}}\left\|e_{s}^{i-1}\right\|_{T_{r}, 0}^{2} .
\end{aligned}
$$

From this one obtains

$$
\begin{aligned}
& \frac{L_{s} \phi}{2} \sum_{T_{r} \in \mathcal{T}}\left\|e_{s}^{i}\right\|_{T_{r}, 0}^{2}+\frac{\Delta t L_{s, T} \phi}{2} \sum_{T_{r} \in \mathcal{T}}\left\|\partial^{-} e_{s}^{i}\right\|_{T_{r}, 0}^{2} \\
+ & {\left[\frac{l_{p_{c, e q}}^{2} \phi}{2 L_{s}}-\Delta t C\left(2+\frac{\theta^{2}}{\left|F_{r}\right|^{2}}\right)\right] \sum_{T_{r} \in \mathcal{T}}\left\|e_{s}^{i-1}\right\|_{T_{r}, 0}^{2}+\frac{l_{T}^{2} \Delta t \phi}{2 L_{s, T}} \sum_{T_{r} \in \mathcal{T}}\left\|\partial^{-} e_{s}^{i-1}\right\|_{T_{r}, 0}^{2} } \\
+ & \frac{\Delta t \underline{\lambda_{n}}}{2} \sum_{T_{r} \in \mathcal{T}}\left\|K^{\frac{1}{2}} \nabla e_{p_{n}}^{i}\right\|_{T_{r}, 0}^{2}+\Delta t\left(\frac{\sigma_{n}}{2}-(1-\theta)^{2} \frac{3{\overline{\lambda_{n}}}^{2} C_{t}^{2} \tilde{C}^{2}}{2 \underline{\lambda_{n}}}\right) \sum_{F_{r} \in \mathcal{F}} \frac{f\left(k_{p}\right)}{\left|F_{r}\right|}\left\|\llbracket e_{p_{n}}^{i} \rrbracket\right\|_{F_{r}, 0}^{2} \\
+ & \frac{\Delta t \underline{\lambda_{w}}}{2} \sum_{T_{r} \in \mathcal{T}}\left\|K^{\frac{1}{2}} \nabla e_{p_{w}}^{i}\right\|_{T_{r}, 0}^{2}+\Delta t\left(\frac{\sigma_{w}}{2}-(1-\theta)^{2} \frac{3{\overline{\lambda_{w}}}^{2} C_{t}^{2} \tilde{C}^{2}}{2}\right) \sum_{F_{r} \in \mathcal{F}} \frac{f\left(k_{p}\right)}{\left|F_{r}\right|}\left\|\llbracket e_{p_{w}}^{i}\right\|_{F_{r}, 0}^{2} \\
\leq & \frac{L_{s} \phi}{2} \sum_{T_{r} \in \mathcal{T}}\left\|e_{s}^{i-1}\right\|_{T_{r}, 0}^{2}+\frac{\Delta t L_{s, T} \phi}{2} \sum_{T_{r} \in \mathcal{T}}\left\|\partial^{-} e_{s}^{i-1}\right\|_{T_{r}, 0}^{2},
\end{aligned}
$$

which can be reformulated as

$$
\begin{aligned}
& \frac{L_{s} \phi}{2} \sum_{T_{r} \in \mathcal{T}}\left\|e_{s}^{i}\right\|_{T_{r}, 0}^{2}+\frac{\Delta t L_{s, T} \phi}{2} \sum_{T_{r} \in \mathcal{T}}\left\|\partial^{-} e_{s}^{i}\right\|_{T_{r}, 0}^{2} \\
& +\frac{\Delta t \underline{\lambda_{n}}}{2} \sum_{T_{r} \in \mathcal{T}}\left\|K^{\frac{1}{2}} \nabla e_{p_{n}}^{i}\right\|_{T_{r}, 0}^{2}+\Delta t\left(\frac{\sigma_{n}}{2}-(1-\theta)^{2} \frac{3{\overline{\lambda_{n}}}^{2} C_{t}^{2} \tilde{C}^{2}}{2 \underline{\lambda_{n}}}\right) \sum_{F_{r} \in \mathcal{F}} \frac{f\left(k_{p}\right)}{\left|F_{r}\right|}\left\|\llbracket e_{p_{n}}^{i} \rrbracket\right\|_{F_{r}, 0}^{2} \\
& \quad+\frac{\Delta t \underline{\lambda_{w}}}{2} \sum_{T_{r} \in \mathcal{T}}\left\|K^{\frac{1}{2}} \nabla e_{p_{w}}^{i}\right\|_{T_{r}, 0}^{2}+\Delta t\left(\frac{\sigma_{w}}{2}-(1-\theta)^{2} \frac{3{\overline{\lambda_{w}}}^{2} C_{t}^{2} \tilde{C}^{2}}{2 \underline{\lambda_{w}}}\right) \sum_{F_{r} \in \mathcal{F}} \frac{f\left(k_{p}\right)}{\left|F_{r}\right|}\left\|\llbracket e_{p_{w}}^{i} \rrbracket\right\|_{F_{r}, 0}^{2} \\
& \leq\left[\frac{L_{s} \phi}{2}-\left(\frac{l_{p_{c, e q}}^{2} \phi}{2 L_{s}}-\Delta t C\left(2+\frac{\theta^{2}}{\left|F_{r}\right|^{2}}\right)\right)\right] \sum_{T_{r} \in \mathcal{T}}\left\|e_{s}^{i-1}\right\|_{T_{r}, 0}^{2} \\
& \quad+\left(\frac{\Delta t L_{s, T} \phi}{2}-\frac{l_{T}^{2} \Delta t \phi}{2 L_{s, T}}\right) \sum_{T_{r} \in \mathcal{T}}\left\|\partial^{-} e_{s}^{i-1}\right\|_{T_{r}, 0}^{2} .
\end{aligned}
$$


For $\Delta t$ small enough, this leads to a contraction for the terms $\sum_{T_{r} \in \mathcal{T}}\left\|e_{s}^{i}\right\|_{T_{r}, 0}^{2}$ and $\sum_{T_{r} \in \mathcal{T}}\left\|\partial^{-} e_{s}^{i}\right\|_{T_{r}, 0}^{2}$, which concludes the proof.

\section{Remark 2}

To obtain the contraction it is requires that the time-step is chosen s.t.

$$
\Delta t<\frac{l^{2} p_{c, e q} \phi}{2 L_{s} C\left(2+\frac{\theta^{2}}{\left|F_{\min }\right|^{2}}\right)},
$$

where $\left|F_{\min }\right|$ is the measure of the smallest face. This restriction is milder when compared to the typical stability conditions imposed for explicit methods (like IMPES), or for the Newton method (see e.g. [32] for the analysis for a simplified two-phase model). Moreover, for the IIPG (incomplete interior penalty Galerkin) method, in which $\theta=0$, the constraint on the time-step does not depend on the mesh size at all and is similar to the one for the $L$-method for standard, equilibrium two-phase flows when $\tau=0$, see [30].

\section{Remark 3}

To guarantee the convergence, the parameters $L_{s}$ and $L_{s, T}$ must satisfy (23). For degenerate problems, if e.g. the equilibrium capillary pressure function is not Lipschitz one needs to regularize first the problem in order to ensure the convergence of the scheme.

\section{Remark 4}

The convergence result can be extended to conforming discretizations like finite elements, when the approximation lies in $W^{1,2}(\Omega)$. On can carry out the similar steps as above, but now jumps and averages over faces do not appear anymore. As in the IIPG, in this case the restriction on the time step does not depend on the mesh size, leading to results that are similar to the ones in [30].

\section{NUMERICAL EXPERIMENTS}

Having proved rigorously the convergence of the iteration scheme, we now test numerically its effectiveness. To do so, two test problems are considered. In both cases a Brooks-Corey parametrization is assumed (see e.g. [15, 26]). For the first problem an analytical solution can be obtained and is used for estimating the errors explicitly. The second uses realistic parameters and nonlinearities and is defined in a heterogeneous porous medium. The focus here is on the convergence of the proposed linearization scheme, and comparison with Newton's method. As mentioned again, the convergence of the dG/backward Euler discretization is proved in [16]. For the implementation of the numerical scheme, we used DUNE-PDELab [1, 2].

\subsection{Example 1: A problem with known analytical solution}

We consider the domain $\Omega=(0,1) \times(0,1) \subset \mathbb{R}^{2}$ and the time interval $[0,1]$. The other parameters are listed in Table I. 
Table I. Example 1 - Properties

\begin{tabular}{lll}
\hline Phase Properties & \\
\hline water dynamic viscosity & $\mu_{w}\left[\frac{\mathrm{kg}}{\mathrm{ms}}\right]$ & 1 \\
oil dynamic viscosity & $\mu_{n}\left[\frac{\mathrm{kg}}{\mathrm{ms}}\right]$ & 1 \\
water density & $\rho_{w}\left[\frac{\mathrm{kg}}{\mathrm{m}^{3}}\right]$ & 1 \\
oil density & $\rho_{n}\left[\frac{\mathrm{kg}}{\mathrm{m}^{3}}\right]$ & 1 \\
\hline Hydraulic Properties & & \\
\hline absolute permeability & $K\left[\mathrm{~m}^{2}\right]$ & 1 \\
residual water saturation & $S_{r w}$ & 0 \\
residual oil saturation & $S_{r n}$ & 0 \\
porosity & $\varphi$ & 0.4 \\
damping coefficient & $\tau[\mathrm{Pa} \cdot s]$ & 1 \\
\hline Brooks-Correy Parameters & & \\
\hline entry pressure & $p_{d}[\mathrm{~Pa}]$ & 1 \\
pore size distribution index & $\lambda$ & 2 \\
\hline
\end{tabular}

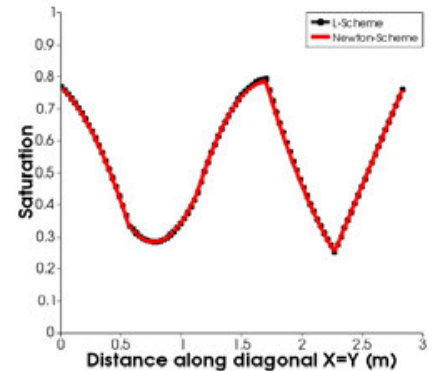

(a) $s_{w}$ at $t=5$ for the mesh size $h=0.4$

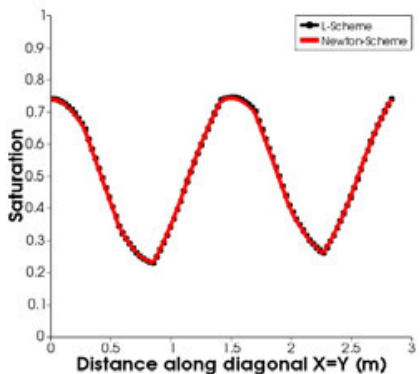

(b) $s_{w}$ at $t=5$ for the mesh size $h=0.2$

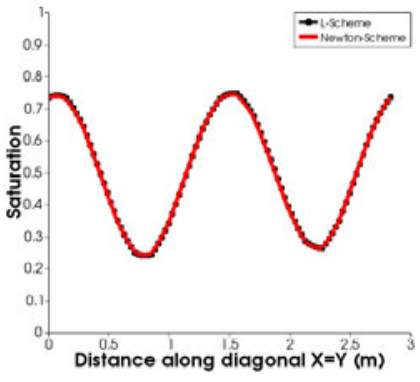

(c) $s_{w}$ at $t=5$ for the mesh size $h=0.1$

Figure 1. Problem 1 - Comparison of the Newton-scheme and the L-scheme for various meshes.

The right hand sides (sources) in the equations and the boundary and initial conditions are chosen such that the following are the exact solution of the model

$$
\begin{aligned}
& p_{n}(t, x, y)=\frac{1}{4} \cos ((x+y) \pi-t)+\frac{1}{2}, \\
& S_{w}(t, x, y)=\frac{1}{4} \sin ((x+y) \pi-t)+\frac{1}{2} \\
& p_{c}(t, x, y)=p_{c, e q}\left(S_{w}(t, x, y)\right)-\partial_{t} T\left(S_{w}(t, x, y)\right) .
\end{aligned}
$$

We chose $\theta=0$ and the penalty parameters as $\sigma_{w}=\sigma_{n}=10$. We set

$$
T\left(S_{w}(t, x, y)\right):=\tau S_{w}(t, x, y)
$$

which corresponds to a constant damping factor $\tau$. The initial mesh, consisting of five elements in each direction, is refined successively by doubling the number of elements after each simulation. We first solve the nonlinear system by using the Newton-scheme to get a reference simulation. Then we solve the same problem using the L-scheme chosing $L_{s}=0.15$. In both cases we use a time-step size of $\Delta t=0.1$. 


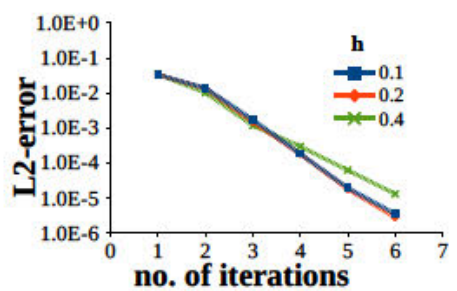

(a) $L^{2}$ error in $p_{n}$.

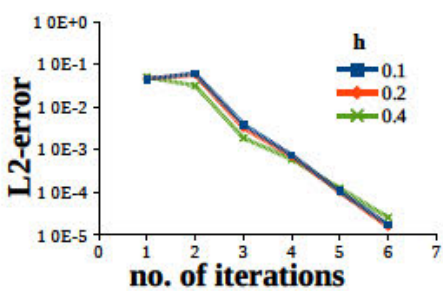

(b) $L^{2}$ error in $p_{c}$.

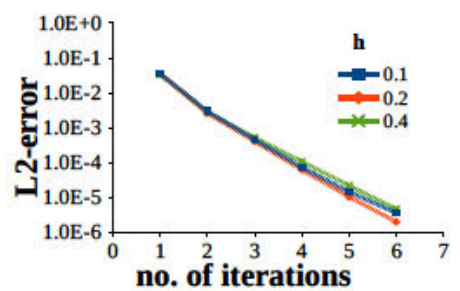

(c) $L^{2}$ error in $s_{w}$.

Figure 2. Example 1 - Error decay for the $L$-scheme at $t=5$ and on various meshes.

Table II. Example 2 - Properties

\begin{tabular}{|c|c|c|c|}
\hline & & case $\mathrm{A}$ & case B \\
\hline \multicolumn{4}{|l|}{ Phase Properties } \\
\hline water dynamic viscosity & $\mu_{w}\left[\frac{\mathrm{kg}}{2}\right]$ & $10^{-3}$ & $10^{-3}$ \\
\hline oil dynamic viscosity & $\mu_{n}\left[\frac{\mathrm{kg}}{\mathrm{ms}}\right]$ & $10^{-3}$ & $10^{-3}$ \\
\hline water density & $\rho_{w}\left[\frac{\mathrm{kg}}{\mathrm{m}^{3}}\right]$ & $10^{3}$ & $10^{3}$ \\
\hline oil density & $\rho_{n}\left[\frac{\mathrm{kg}}{\mathrm{m}^{3}}\right]$ & $1.623 \cdot 10^{3}$ & $1.623 \cdot 10^{3}$ \\
\hline \multicolumn{4}{|l|}{ Hydraulic Properties } \\
\hline absolute permeability & $K\left[m^{2}\right]$ & $10^{-11}$ & $\begin{cases}10^{-11} & \text { if } x<0.5, y<0.5 \\
10^{-11} & \text { if } x>0.5, y>0.5 \\
10^{-12} & \text { elsewhere }\end{cases}$ \\
\hline residual water saturation & $S_{r w}$ & 0 & 0 \\
\hline residual oil saturation & $S_{r n}$ & 0 & 0 \\
\hline porosity & $\varphi$ & 0.2 & 0.2 \\
\hline damping coefficient & $\tau[P a \cdot s]$ & $10^{5}$ & $10^{5}$ \\
\hline \multicolumn{4}{|l|}{ Brooks-Correy Parameters } \\
\hline entry pressure & $p_{d}[P a]$ & $2.5 \cdot 10^{3}$ & $2.5 \cdot 10^{3}$ \\
\hline pore size distribution index & $\lambda$ & 2 & 2 \\
\hline
\end{tabular}

In Figures $1 \mathrm{a}, 1 \mathrm{~b}$, and $1 \mathrm{c}$, we compare the solution provided by the Newton scheme and by the $\mathrm{L}$-scheme at $t=5$. As one can see, the solutions are difficult to distinguish.

At eacht time step, the Newton scheme takes about $3-4$ iterations to converge up to a tolerance error of $10^{-6}$. Since the $L$-scheme converges linearly, one would expect more iterations to obtain a comparable error in the solution. However, as shown in Figures 2a, 2b, and 2c, $6 L$-iterations are sufficient to achieve a similar accuracy. We point out that the number of iteration is not depending on the mesh size.

\subsection{Example 2: Inflow problem in homogeneous and heterogenous permeability fields}

In this example, we consider an inflow problem in the domain $\Omega=(0 \mathrm{~m}, 1 \mathrm{~m}) \times(0 \mathrm{~m}, 1 \mathrm{~m})$ and over the time interval $[0 s, 2500 s]$. Also, the parameters and nonlinearities are realistic and two situations are considered: a homogenous medium (case A) and a non-homogenous (case B) one. The properties for both cases are given in Table II. The initial and the boundary conditions are given in Table III. 
Table III. Example 2 - Boundary and initial conditions

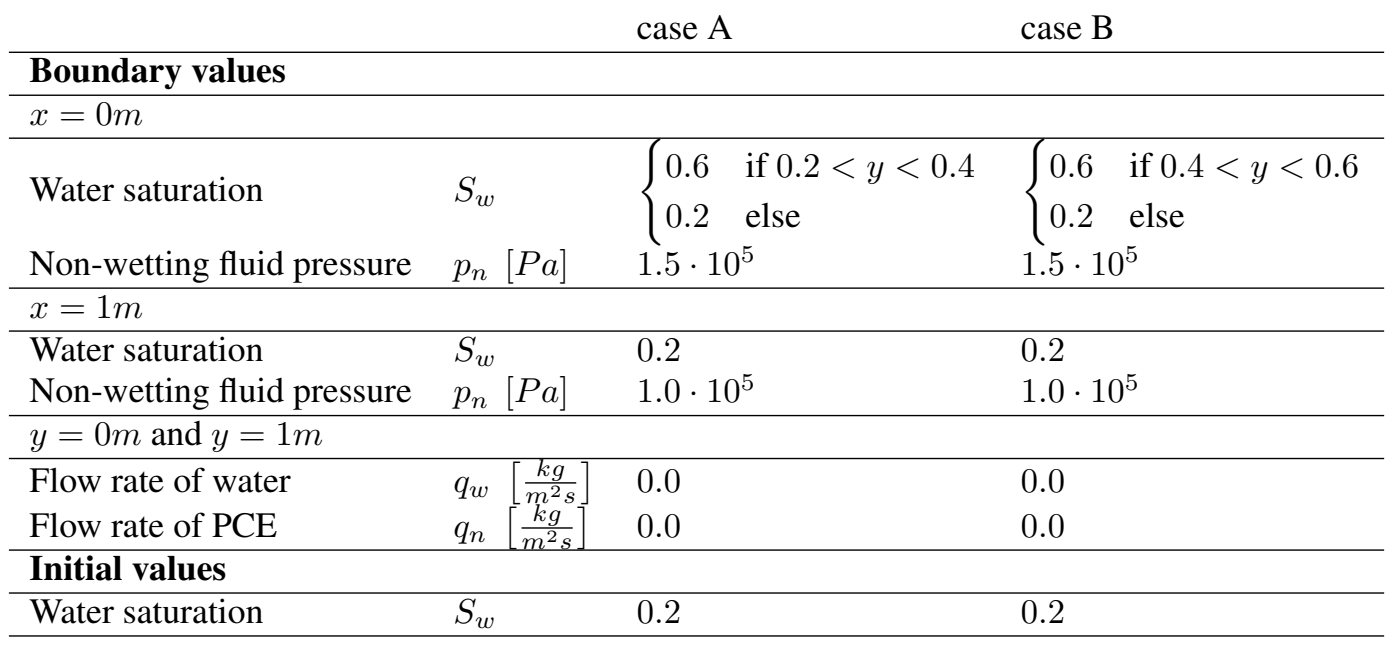

For the $L$-scheme we take $L_{s}=0.1$. Since the dynamic term is linear, no additional linearization is needed. The time-step is chosen as $d t=10 \mathrm{~s}$, and the domain is discretized by taking $50 \times 50=$ 2500 elements. We again chose $\theta=0$ and $\sigma_{w}=\sigma_{n}=10$. As displayed in Figure 3a, in case A a straight finger is being formed, propagating with the flow (from left to right). For case B, the choice in the absolute permeability $K$ leads to a preferential flow through the highly permeable medium, and a flow path is formed from the lower left to the upper right quadrant. This can be seen in Figure $3 b$.

For both cases the L-scheme performs well and leads to results as expected.

\section{SUMMARY}

We considered a non-standard model for two-phase flow in porous media. The fluids are assumed to be incompressible and immiscible and the porous matrix is non-deformable. The model includes a dynamic term in the phase pressure difference - saturation relationship. We propose a discretization scheme based on the discontinuous Galerkin method in space and on backward Euler in time. A robust linear scheme is proposed to solve the nonlinear problems appearing at each time step. This scheme does not require computing any derivatives. It converges linearly, however, for any starting point. The convergence of the scheme is proved rigorously. Finally, numerical results are presented, confirming the theoretical findings.

\section{ACKNOWLEDGEMENT}

Stefan Karpinski wants to thank his employers, Dr. Roman Rojko and Dr. Stefan Zaprianov from ESPRiT Engineering GmbH for their mentorship and support. Part of this work was done during F.A. Radu's sabbatical in Eindhoven, we acknowledge for this the support of Meltzer foundation, of University of Bergen and the NWO Visitors Grant 040.11.499. I.S. Pop is supported by the Research Foundation Flanders FWO through the the Odysseus programme grant G0G1316N. Finally, F. A. Radu and I. S. Pop acknowledge the support of Statoil through the Akademia agreement. 


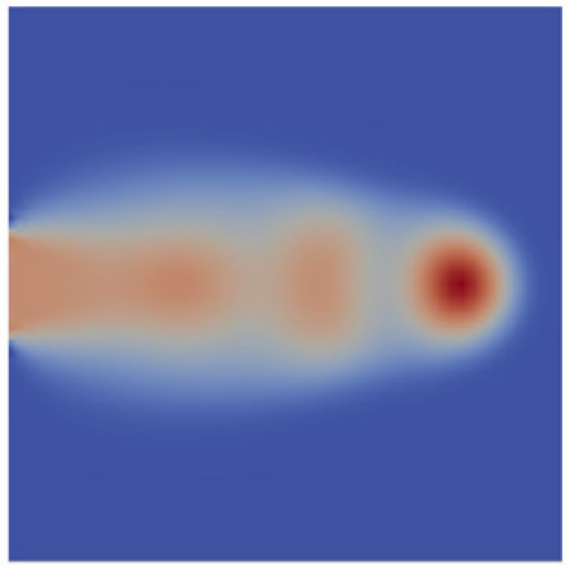

Sw

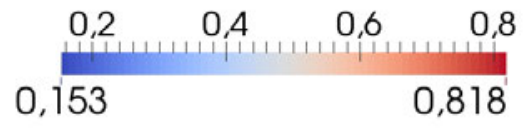

(a) $S_{w}$ at $t=1500 s$ for case A.

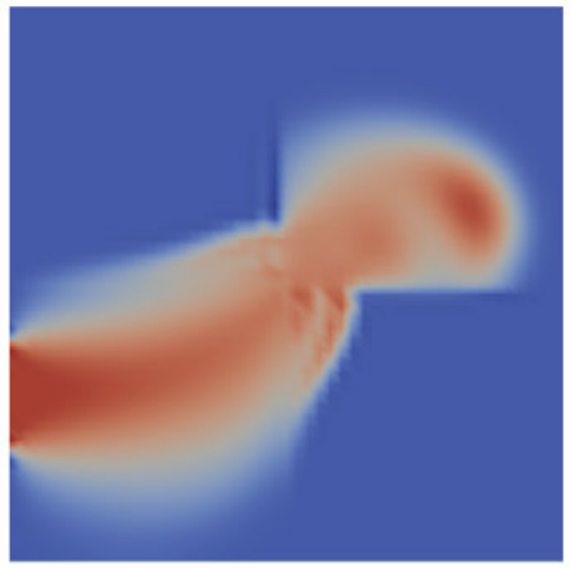

Sw

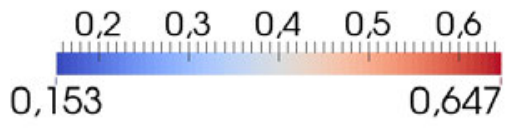

(b) $S_{w}$ at $t=2500 \mathrm{~s}$ for case B.

Figure 3. Example 2 - Saturation profiles at time $t=2500$ s for a homogeneus medium (left) and a heterogeneous one (right).

\section{References}

1. P. Bastian, M. Blatt, A. Dedner, Ch. Engwer, J. Fahlke, C. Gräser, R. Klöfkorn, M. Nolte, M. Ohlberger, and O. Sander. DUNE Web page, 2011. http://www.dune-project.org.

2. P. Bastian, F. Heimann, and S. Marnach. Generic implementation of finite element methods in the distributed and unified numerics environment (dune). Kybernetika, 46(2):294-315, 2010.

3. L. Bergamaschi and M. Putti. Mixed finite elements and newton-type linearizations for the solution of richards' equation. Int. J. Num. Meth. Engng., 45(1):1025-1046, 1999.

4. X. Cao and I. S. Pop. Degenerate two-phase porous media flow model with dynamic capillarity. J. Differential Equations, 260(3):2418-2456, 2016.

5. X. Cao and I.S. Pop. Two-phase porous media flows with dynamic capillary effects and hysteresis : uniqueness of weak solutions. Computers and Mathematics with Applications, 69(2015)(7):688-695, 2015.

6. M. Celia, E. Bouloutas, and R. R. Zarba. A general mass-conservative numerical solution for the unsaturated flow equation. Water Resour. Res., 26:1483-1496, 1990.

7. D.A. Di Pietro and A. Ern. Discrete functional analysis tools for discontinuous galerkin methods with application to the incompressible navier-stokes equations. Mathematics of Computation, 79(271):13031330, 2010.

8. D.A. Di Pietro and A. Ern. Mathematical Aspects of Discontinuous Galerkin Methods. Springer, 2012.

9. D.A. DiCarlo. Experimental measurements of saturation overshoot on infiltration. Water Resources Research, 40(4), 2004. W04215.

10. H.-J.G. Diersch, V. Clausnitzer, V. Myrnyy, R. Rosati, M. Schmidt, H. Beruda, B.J. Ehrnsperger, and R. Virgilio. Modeling unsaturated flow in absorbent swelling porous media: Part 1. theory. Transport in Porous Media, 83(3):437-464, 2010

11. Y. Epshteyn and B. Riviere. Analysis of discontinuous galerkin methods for incompressible two-phase flow. Journal of Computational and Applied Mathematics, 225(2):487 - 509, 2009.

12. S.M. Hassanizadeh and A.Yu. Beliaev. A theoretical model of hysteresis and dynamic effects in the capillary relation for two-phase flow in porous media. Transport in Porous Media, 43(3):487-510, 2001.

13. S.M. Hassanizadeh, M. Celia, and H.K. Dahle. Dynamic efect in the capillary pressure-saturation relationship and its impacts on unsaturated flow. Vadose Zone Journal, 1(1):38-57, 2002.

14. S.M. Hassanizadeh and W.G. Gray. Thermodynamic basis of capillary pressure in porous media. Water Resources Research, 29(10):3389-3405, 1993. 
15. R. Helmig. Multiphase Flow and Transport Processes in the Subsurface: A Contribution to the Modeling of Hydrosystems. Environmental engineering. Springer, 1997.

16. S. Karpinski and I.S. Pop. Analysis of an interior penalty discontinuous galerkin scheme for two phase flow in porous media with dynamic capillarity effects. Numer. Math., 2016.

17. J. Koch, A. Rätz, and B. Schweizer. Two-phase flow equations with a dynamic capillary pressure. European Journal of Applied Mathematics, 24:49-75, 22013.

18. J. Kou and S. Sun. A new treatment of capillarity to improve the stability of impes two-phase ow formulation. Computers and Fluids, 39:1923-1931, 2010.

19. J. Kou and S. Sun. On iterative impes formulation for two phase ow with capillarity in heterogeneous porous media. Inter. J. of Numer. Anal. and Modeling Series B, pages 20-40, 2010.

20. S. Kraeutle. The semismooth newton method for multicomponent reactive transport with minerals. Adv. Water Resources, 34(5):137-151, 2011.

21. F. Lehmann and $\mathrm{Ph}$. Ackerer. Comparison of iterative methods for improved solutions of the fluid flow equation in partially saturated porous media. Transport in porous media, 31:275-292, 1998.

22. Buyang Li. Maximum-norm stability and maximal $L^{p}$ regularity of FEMs for parabolic equations with Lipschitz continuous coefficients. Numer. Math., 131(3):489-516, 2015.

23. F. List and F. A. Radu. A study on iterative methods for solving Richards' equation. Comput. Geosci., 20:341-353, 2016.

24. R. Neumann, P. Bastian, and O. Ippisch. Modeling and simulation of two-phase two-component flow with disappearing nonwetting phase. Computational Geosciences, 17:139-149, 2013.

25. J. A. Nitsche and Mary F. Wheeler. $L_{\infty}$-boundedness of the finite element Galerkin operator for parabolic problems. Numer. Funct. Anal. Optim., 4(4):325-353, 1981/82.

26. J.M. Nordbotten and M.A. Celia. Geological Storage of CO2: Modeling Approaches for Large-Scale Simulation. Wiley, 2011.

27. E.J. Park. Mixed finite elements for nonlinear second-order elliptic problems. SIAM J. Numer. Anal., 32:865885, 1995.

28. I.S. Pop, F.A. Radu, and P. Knabner. Mixed finite elements for the richards' equations: linearization procedure. $J$. Comput. Appl. Math., 168(1-2):365-373, 2004.

29. F.A. Radu, J.M. Nordbotten, I.S. Pop, and K. Kumar. A convergent mass conservative numerical scheme based on mixed finite elements for two-phase flow in porous media. arXiv, 1512.08387:1 - 32, 2015.

30. F.A. Radu, J.M. Nordbotten, I.S. Pop, and K. Kumar. A robust linearization scheme for finite volume based discretizations for simulation of two-phase flow in porous media. Journal of Computational and Applied Mathematics, 289(0):134 - 141, 2015.

31. F.A. Radu and I.S. Pop. Mixed finite element discretization and newton iteration for a reactive contaminant transport model with nonequilibrium sorption: convergence analysis and error estimates. Comput. Geosci., 15(3):431-450, 2011.

32. F.A. Radu, I.S. Pop, and P. Knabner. On the convergence of the newton method for the mixed finite element discretization of a class of degenerate parabolic equation. In Numerical Mathematics and Advanced Applications. A. Bermudez de Castro et al. (editors), Springer., pages 1194-1200, 2006.

33. B. Riviere, M.F. Wheeler, and V. Girault. A priori error estimates for finite element methods based on discontinuous approximation spaces for elliptic problems. SIAM J. Numer. Anal., 39(3):902-931, March 2001.

34. M. Slodička. A robust and efficient linearization scheme for doubly nonlinear and degenerate parabolic problems arising in flow in porous media. SIAM J. Sci. Comput., 23(5):1593-1614, 2002.

35. T. Warburton and J.S. Hesthaven. On the constants in hp-finite element trace inverse inequalities. Computer Methods in Applied Mechanics and Engineering, 192(25):2765 - 2773, 2003.

36. W.A. Yong and I.S. Pop. A numerical approach to porous medium equations. Preprint, IWR Heidelberg, 95-50, 1996. 


\section{$\underset{\rightarrow \text { universiteit }}{\text { unselt }}$}

\section{UHasselt Computational Mathematics Preprint Series}

UP-16-01 Jochen Schütz and Vadym Aizinger, A hierarchical scale separation approach for the hybridized discontinuous Galerkin method, 2016

UP-16-02 Klaus Kaiser, Jochen Schütz, Ruth Schöbel and Sebastian Noelle, A new stable splitting for the isentropic Euler equations, 2016

UP-16-03 Sergey Alyaev, Eirik Keilegavlen, Jan Martin Nordbotten, Iuliu Sorin Pop, Fractal structures in freezing brine, 2016

UP-16-04 Florin A. Radu, Kundan Kumar, Jan Martin Nordbotten, Iuliu Sorin Pop, A robust, mass conservative scheme for two-phase flow in porous media including Hölder continuous nonlinearities, 2016

UP-16-05 Stefan Karpinski, Iuliu Sorin Pop, Florin A. Radu, Analysis of a linearization scheme for an interior penalty discontinuous Galerkin method for two phase flow in porous media with dynamic capillarity effects, 2016 